TRANSACTIONS OF THE

AMERICAN MATHEMATICAL SOCIETY

Volume 363, Number 6, June 2011, Pages 2907-2937

S 0002-9947(2011)05195-0

Article electronically published on January 27, 2011

\title{
EINSTEIN AND CONFORMALLY FLAT CRITICAL METRICS OF THE VOLUME FUNCTIONAL
}

\author{
PENGZI MIAO AND LUEN-FAI TAM
}

\begin{abstract}
Let $R$ be a constant. Let $\mathcal{M}_{\gamma}^{R}$ be the space of smooth metrics $g$ on a given compact manifold $\Omega^{n}(n \geq 3)$ with smooth boundary $\Sigma$ such that $g$ has constant scalar curvature $R$ and $\left.g\right|_{\Sigma}$ is a fixed metric $\gamma$ on $\Sigma$. Let $V(g)$ be the volume of $g \in \mathcal{M}_{\gamma}^{R}$. In this work, we classify all Einstein or conformally flat metrics which are critical points of $V(\cdot)$ in $\mathcal{M}_{\gamma}^{R}$.
\end{abstract}

\section{INTRODUCTION}

In [11, the authors studied variational properties of the volume functional, constraint to the space of metrics of constant scalar curvature with a prescribed boundary metric, on a given compact manifold with boundary. More precisely, let $\Omega^{n}$ $(n \geq 3)$ be a connected, compact $n$-dimensional manifold with smooth boundary $\Sigma$ with a fixed boundary metric $\gamma$. Let $R$ be a constant. Let $\mathcal{M}_{\gamma}^{R}$ be the space of metrics on $\Omega$ which have constant scalar curvature $R$ and have induced metric on $\Sigma$ given by $\gamma$. It was proved in [11] that if $g \in \mathcal{M}_{\gamma}^{R}$ is an element such that the first Dirichlet eigenvalue of $(n-1) \Delta_{g}+R$ on $\Omega$ is positive, then $\mathcal{M}_{\gamma}^{R}$ has a manifold structure near $g$. Hence one can study variation of the volume functional near $g$ in $\mathcal{M}_{\gamma}^{R}$. The authors [11] proved that: $g$ is a critical point of the usual volume functional $V(\cdot)$ in $\mathcal{M}_{\gamma}^{R}$ if and only if there is a function $\lambda$ on $\Omega$ such that $\lambda=0$ at $\Sigma$ and

$$
-\left(\Delta_{g} \lambda\right) g+\nabla_{g}^{2} \lambda-\lambda \operatorname{Ric}(g)=g \text { on } \Omega,
$$

where $\Delta_{g}, \nabla_{g}^{2}$ are the Laplacian, Hessian operators with respect to the metric $g$ and $\operatorname{Ric}(g)$ is the Ricci curvature of $g$.

The above result suggests the following definition:

Definition 1.1. Given a compact manifold $\Omega$ with smooth boundary, we say a metric $g$ on $\Omega$ is a critical metric if $g$ satisfies (1.1) for some function $\lambda$ that vanishes on the boundary of $\Omega$.

It was shown in 11] that equation (1.1) alone indeed implies that $g$ has constant scalar curvature. Hence, a critical metric necessarily has constant scalar curvature.

Received by the editors January 7, 2009.

2010 Mathematics Subject Classification. Primary 53C20; Secondary 58JXX.

The first author was supported in part by Australian Research Council Discovery Grant \#DP0987650.

The second author was supported in part by Hong Kong RGC General Research Fund \#CUHK403108.

(C)2011 American Mathematical Society Reverts to public domain 28 years from publication 2907 
A natural question is to characterize critical metrics. We have the following results from [11:

(i) If $\Omega$ is a bounded domain with smooth boundary in a simply connected space form $\mathbb{R}^{n}, \mathbb{H}^{n}$ or $\mathbb{S}^{n}$, then the corresponding space form metric is a critical metric on $\Omega$ if and only if $\Omega$ is a geodesic ball (if $\Omega \subset \mathbb{S}^{n}$, one assumes $\left.V(\Omega)<\frac{1}{2} V\left(\mathbb{S}^{n}\right)\right)$.

(ii) If $g$ is a critical metric with zero scalar curvature on a compact manifold $\Omega$ such that the boundary of $(\Omega, g)$ is isometric to a geodesic sphere $\Sigma_{0}$ in $\mathbb{R}^{n}$, then $V(g) \geq V_{0}$, where $V_{0}$ is the Euclidean volume enclosed by $\Sigma_{0}$. Moreover, $V(g)=V_{0}$ if and only if $(\Omega, g)$ is isometric to a Euclidean geodesic ball.

These results suggest that critical metrics with a prescribed boundary metric seem to be rather rigid. For instance, we want to know if there exist non-constant sectional curvature critical metrics on a compact manifold whose boundary is isometric to a standard round sphere. If yes, what can we say about the structure of such metrics?

In this paper, we study this rigidity question under certain additional assumptions: We assume the manifold is Einstein or is conformally flat. Since space forms are both Einstein and conformally flat, these considerations are natural steps to follow the results in [11. Our study of conformally flat critical metrics are also motivated by the work of Kobayashi and Obata [ 8,9 .

The first result we obtain in this work is the following:

Theorem 1.1. Let $(\Omega, g)$ be a connected, compact, Einstein manifold with a smooth boundary $\Sigma$. Suppose the metric $g$ is a critical metric. Then $\left(\Omega^{n}, g\right)$ is isometric to a geodesic ball in a simply connected space form $\mathbb{R}^{n}, \mathbb{H}^{n}$ or $\mathbb{S}^{n}$.

To understand conformally flat critical metrics, we first construct explicit examples of critical metrics which are in the form of warped products. It is interesting to note that those examples include the usual spatial Schwarzschild metrics and AdsSchwarzschild metrics restricted to certain domains containing their horizon and bounded by two spherically symmetric spheres (see Corollaries 3.1 and 3.2). Then we show that any conformally flat, non-Einstein, critical metric is either one of the warped products we construct or it is covered by such a metric. More precisely, we have:

Theorem 1.2. Let $\left(\Omega^{n}, g\right)$ be a connected, compact, conformally flat manifold with a smooth boundary $\Sigma$. Suppose the metric $g$ is a critical metric and the first Dirichlet eigenvalue of $(n-1) \Delta_{g}+R$ is non-negative, where $R$ is the scalar curvature of $g$.

(i) If $\Sigma$ is disconnected, then $\Sigma$ has exactly two connected components, and $(\Omega, g)$ is isometric to $\left(I \times N, d s^{2}+r^{2} h\right)$ where $I$ is a finite interval in $\mathbb{R}^{1}$ containing the origin $0,(N, h)$ is a closed manifold with constant sectional curvature $\kappa_{0}, r$ is a positive function on I satisfying $r^{\prime}(0)=0$ and

$$
r^{\prime \prime}+\frac{R}{n(n-1)} r=a r^{1-n}
$$

for some constant $a>0$, and the constant $\kappa_{0}$ satisfies

$$
\left(r^{\prime}\right)^{2}+\frac{R}{n(n-1)} r^{2}+\frac{2 a}{n-2} r^{2-n}=\kappa_{0} .
$$


(ii) If $\Sigma$ is connected, then $(\Omega, g)$ is either isometric to a geodesic ball in a simply connected space form $\mathbb{R}^{n}, \mathbb{H}^{n}$, or $\mathbb{S}^{n}$, or $(\Omega, g)$ is covered by one of the above mentioned warped products in (i) with a covering group $\mathbb{Z}_{2}$.

It follows from Theorem 1.2 that if $g$ is a conformally flat critical metric on a simply connected manifold $\Omega$ such that the boundary of $(\Omega, g)$ is isometric to a standard round sphere, then $(\Omega, g)$ is isometric to a geodesic ball in $\mathbb{R}^{n}, \mathbb{H}^{n}$ or $\mathbb{S}^{n}$.

The organization of the paper is as follows. In Section 2, we consider critical metrics which are Einstein. We prove that compact manifolds with critical Einstein metrics are geodesic balls in simply connected space forms. In Section 3, we construct critical metrics which can be written as a warped product or the quotient of a warped product. In particular, we obtain non-Einstein critical metrics whose boundary is a standard round sphere and examples of critical metrics whose boundary is disconnected. In Section 4, we classify all conformally flat critical metrics. We prove that they are exactly the metrics constructed in Section 2. For completeness and easy reference, we include an appendix on estimates of graphical representation of hypersurfaces with bounded second fundamental form, which is needed in Section 4. All manifolds considered in this paper are assumed to be connected with dimension $n \geq 3$.

\section{Critical Einstein metrics}

Let $(M, g)$ be an Einstein manifold with or without boundary. We normalize $g$ so that $\operatorname{Ric}(g)=(n-1) \kappa g$, where $\kappa=0,1$, or -1 . Suppose there is a non-constant function $\lambda$ on $M$ satisfying

$$
-\left(\Delta_{g} \lambda\right) g+\nabla_{g}^{2} \lambda-\lambda \operatorname{Ric}(g)=g .
$$

We will prove in Theorem 2.1 that, if $M$ is connected, compact with non-empty boundary on which $\lambda$ is zero, then $(M, g)$ is isometric to a geodesic ball in $\mathbb{R}^{n}, \mathbb{H}^{n}$ or $\mathbb{S}^{n}$. In Theorem 2.2, we will also classify those $(M, g)$ that are complete without boundary.

We note that all geodesics in this section are assumed to be parametrized by arc-length.

Lemma 2.1. Let $(M, g)$ and $\lambda$ be given as above. Suppose there exists $p \in M$ such that $\nabla \lambda(p)=0$. Then the following are true:

(i) Along a geodesic $\alpha(s)$ emanating from $p$, we have:

(a) if $\kappa=0$, then

$$
\lambda(\alpha(s))=-\frac{1}{2(n-1)} s^{2}+\lambda(p)
$$

(b) $\kappa=1$, then

$$
\lambda(\alpha(s))=\left(\lambda(p)+\frac{1}{n-1}\right) \cos s-\frac{1}{(n-1)} ;
$$

(c) $\kappa=-1$, then

$$
\lambda(\alpha(s))=\left(\lambda(p)-\frac{1}{n-1}\right) \cosh s+\frac{1}{(n-1)} .
$$

(ii) Suppose $q \in M$ such that there exists a minimizing geodesic $\alpha(s)$ connecting $p$ to $q$. If $\beta(s)$ is another geodesic connecting $p$ to $q$ and $\beta(s)$ has length no greater than $\pi$ if $\kappa=1$, then $\beta(s)$ is also minimizing. 
Proof. As $\operatorname{Ric}(g)=(n-1) \kappa g$, (2.1) is equivalent to

$$
\nabla_{g}^{2} \lambda=\left(-\kappa \lambda-\frac{1}{n-1}\right) g
$$

Hence, $\lambda$ satisfies

$$
\frac{d^{2}}{d s^{2}} \lambda(\alpha(s))=-\kappa \lambda(\alpha(s))-\frac{1}{n-1}
$$

along $\alpha(s)$. From this and the fact $\nabla \lambda(p)=0$, (i) of the lemma follows.

To prove (ii), let $r$ and $l$ be the length of $\alpha(s)$ and $\beta(s)$. By (i) and the fact $\alpha(r)=q=\beta(l)$, we have:

$$
-\frac{1}{2(n-1)} r^{2}+\lambda(p)=-\frac{1}{2(n-1)} l^{2}+l(p)
$$

if $\kappa=0$;

$$
\left(\lambda(p)+\frac{1}{n-1}\right) \cos r-\frac{1}{(n-1)}=\left(\lambda(p)+\frac{1}{n-1}\right) \cos l-\frac{1}{(n-1)}
$$

if $\kappa=1$; and

$$
\left(\lambda(p)-\frac{1}{n-1}\right) \cosh r+\frac{1}{(n-1)}=\left(\lambda(p)-\frac{1}{n-1}\right) \cosh l+\frac{1}{(n-1)}
$$

if $\kappa=-1$. Since $\lambda$ is not identically a constant, we have $\lambda(p)+\frac{1}{n-1} \neq 0$ if $\kappa=1$ and $\lambda(p)-\frac{1}{n-1} \neq 0$ if $\kappa=-1$. In case $\kappa=0$ or -1 , it is then evident that $r=l$. In case $\kappa=1$, we have $\operatorname{Ric}(g)=(n-1) g$, which implies $r \leq \pi$, as $\alpha(s)$ is minimizing. Since $l \leq \pi$ by assumption, we have $r=l$. This shows that $\beta(s)$ is also minimizing.

Lemma 2.2. Let $(M, g)$ and $\lambda$ be given as above. Suppose $\Sigma \subset M$ is a connected, embedded hypersurface on which $\lambda$ equals a constant. Suppose $\nabla \lambda$ never vanishes on $\Sigma$ and let $\nu=\nabla \lambda /|\nabla \lambda|$. Then $|\nabla \lambda|$ is constant on $\Sigma$ and the second fundamental form $A(X, Y)$ of $\Sigma$ with respect to $\nu$ satisfies

$$
A(X, Y)=|\nabla \lambda|^{-1}\left(-\kappa \lambda-\frac{1}{n-1}\right) g(X, Y),
$$

where $X, Y$ are any tangent vectors to $\Sigma$.

Proof. Using the fact that $\lambda$ equals a constant on $\Sigma$, we have

$$
\begin{aligned}
\frac{1}{2} X\left(|\nabla \lambda|^{2}\right) & =\left\langle\nabla_{X}(\nabla \lambda), \nabla \lambda\right\rangle \\
& =|\nabla \lambda|\left\langle\nabla_{X}(\nabla \lambda), \nu\right\rangle \\
& =|\nabla \lambda| \nabla_{g}^{2}(\lambda)(X, \nu)
\end{aligned}
$$

and

$$
\begin{aligned}
A(X, Y) & =\left\langle\nabla_{X} \nu, Y\right\rangle \\
& =|\nabla \lambda|^{-1}\left\langle\nabla_{X}(\nabla \lambda), Y\right\rangle \\
& =|\nabla \lambda|^{-1} \nabla_{g}^{2}(\lambda)(X, Y) .
\end{aligned}
$$

From (2.2), (2.5) and (2.6), we conclude that $X\left(|\nabla \lambda|^{2}\right)=0$ and (2.4) holds. 
Theorem 2.1. Suppose $(\Omega, g)$ is a connected, compact, Einstein manifold with a smooth boundary $\Sigma$. Suppose there is a function $\lambda$ on $\Omega$ such that $\lambda=0$ on $\Sigma$ and

$$
-\left(\Delta_{g} \lambda\right) g+\nabla_{g}^{2} \lambda-\lambda \operatorname{Ric}(g)=g
$$

in $\Omega$. Then $\left(\Omega^{n}, g\right)$ is isometric to a geodesic ball in a simply connected space form $\mathbb{R}^{n}, \mathbb{H}^{n}$ or $\mathbb{S}^{n}$.

Proof. We normalize $g$ such that $\operatorname{Ric}(g)=(n-1) \kappa g$, where $\kappa=0,1$, or -1 . Since $\lambda=0$ on $\Sigma$ and $\lambda$ is not identically zero, there exists an interior point $p \in \Omega$ such that $\nabla \lambda(p)=0$. Let $r_{0}=\operatorname{dist}(p, \Sigma)$, the distance from $p$ to $\Sigma$. Consider the geodesic ball $B_{r_{0}}(p) \subset \Omega$ centered at $p$ with radius $r_{0}$. Then $\partial B_{r_{0}}(p) \cap \Sigma \neq \emptyset$. By Lemma 2.1 we have $\lambda=0$ on $\partial B_{r_{0}}(p)$.

Suppose $\kappa=0$. Then (2.2) implies $\Delta_{g} \lambda<0$. By the maximum principle, we must have $\partial B_{r_{0}}(p) \subset \Sigma$. As $\Omega$ is connected, we have $B_{r_{0}}(p)=\Omega$. Furthermore, the fact $r_{0}=\operatorname{dist}(p, \Sigma)$ implies every geodesic $\alpha(s)$ emanating from $p$ is minimizing on $\left[0, r_{0}\right]$ and every $q \in \Sigma$ can be connected to $p$ by a unique minimizing geodesic with length $r_{0}$. It follows that the exponential map at $p$ is a diffeomorphism onto $B_{r_{0}}(p)=\Omega$. For each $s \in\left(0, r_{0}\right]$, let $\Sigma_{s}$ be the embedded geodesic sphere centered at $p$ of radius $s$. By Lemma 2.1, $\lambda=-\frac{1}{2(n-1)} s^{2}+\lambda(p)$ on $\Sigma_{s}$. In particular, $\nabla \lambda$ does not vanish on $\Sigma_{s}$. Let $H_{s}$ be the mean curvature of $\Sigma_{s}$ w.r.t. the outward unit normal. By Lemma 2.2, we have $H_{s}=\frac{n-1}{s}$. Let $A(s)$ be the areas of $\Sigma_{s}$. Then $\frac{d}{d s} A(s)=\frac{n-1}{s} A(s)$. From this it follows that the volume of $(\Omega, g)$ agrees with the volume of a geodesic ball of radius $r_{0}$ in $\mathbb{R}^{n}$. Since $\operatorname{Ric}(g)=0$, by the Bishop volume comparison theorem [1, we conclude that $(\Omega, g)$ is isometric to a geodesic ball in $\mathbb{R}^{n}$.

Suppose $\kappa=-1$; then (2.2) implies $\Delta_{g} \lambda-n \lambda<0$. The maximum principle can still be applied to show $\partial B_{r_{0}}(p) \subset \Sigma$. Hence we can prove similarly that $(\Omega, g)$ is isometric to a geodesic ball in $\mathbb{H}^{n}$.

Finally, suppose $\kappa=1$. Since $\operatorname{Ric}(g)=(n-1) g$, we have $r_{0} \leq \pi$. In particular, the function $f(s)=\left(\lambda(p)+\frac{1}{n-1}\right) \cos s-\frac{1}{n-1}$ has a nowhere vanishing derivative on $\left(0, r_{0}\right]$. If $\lambda$ never vanishes in the interior of $\Omega$, we can proceed as before to show that $(\Omega, g)$ is isometric to a geodesic ball in $\mathbb{S}^{n}$. In general, let $\Lambda_{0}$ be the set of interior points where $\lambda$ vanishes. Suppose $\nabla \lambda(q)=0$ for some $q \in \Lambda_{0}$. Let $d=$ $\operatorname{dist}(q, \Sigma)$ and let $\beta(s)$ be a geodesic such that $\beta(0)=q$ and $\beta(d) \in \Sigma$. By Lemma 2.1 and the fact $\lambda(q)=0$, we have $\lambda(\beta(s))=\frac{1}{n-1} \cos s-\frac{1}{n-1}$. At $s=d$, we have $\lambda(\beta(d))=0$, hence $\cos d=1$. On the other hand, the fact $\operatorname{Ric}(g)=(n-1) g$ implies $d \leq \pi$, which is a contradiction. Therefore, $\nabla \lambda$ never vanishes at points in $\Lambda_{0}$. In particular, $\Lambda_{0}$ is an embedded hypersurface in $\Omega$.

Let $\Sigma_{1}$ be a connected component of $\Sigma$. At $\Sigma_{1}$, we have $\nabla_{g}^{2} \lambda=-\frac{1}{n-1} g$ by (2.2). As mentioned in [11, this implies that the mean curvature $H$ of $\Sigma_{1}$ (w.r.t. the outward unit normal $\nu$ ) satisfies $H \frac{\partial \lambda}{\partial \nu}=-1$. In particular, $\frac{\partial \lambda}{\partial \nu}$ never vanishes on $\Sigma_{1}$. Suppose $\frac{\partial \lambda}{\partial \nu}<0$ on $\Sigma_{1}$. Since $\lambda=0$ on $\Sigma_{1}$, there exists a connected open set $U_{1}$ in $\Omega$ containing $\Sigma_{1}$ such that $\lambda>0$ on $U_{1} \backslash \Sigma_{1}$. Consider the open set $\Omega^{+}=\{q \in \Omega \mid \lambda(q)>0\}$. Let $\Omega_{1}^{+}$be the connected component of $\Omega^{+}$containing $U_{1} \backslash \Sigma_{1}$. Let $\bar{\Omega}_{1}^{+}$be the closure of $\Omega_{1}^{+}$in $\Omega$. Then $\bar{\Omega}_{1}^{+}$is a compact manifold with smooth non-empty boundary $\partial \bar{\Omega}_{1}^{+}$, moreover, $\lambda>0$ in $\Omega^{+}$and $\lambda=0$ on $\partial \bar{\Omega}_{1}^{+}$. Replacing $\Omega$ by $\bar{\Omega}_{1}^{+}$, we can prove as before that $\left(\bar{\Omega}_{1}^{+}, g\right)$ is isometric to a geodesic ball in $\mathbb{S}^{n}$. In particular, $\partial \bar{\Omega}_{1}^{+}$is connected. Since $\Sigma_{1} \subset \partial \bar{\Omega}_{1}^{+}$, we must 
have $\Sigma_{1}=\partial \bar{\Omega}_{1}^{+}$. Consequently, $\bar{\Omega}_{1}^{+}$is an open set in $\Omega$. Since $\Omega$ is connected, we conclude that $\Omega=\bar{\Omega}_{1}^{+}$and $(\Omega, g)$ is isometric to a geodesic ball in $\mathbb{S}^{n}$. The case $\frac{\partial \lambda}{\partial \nu}>0$ on $\Sigma_{1}$ can be proved similarly by considering $\Omega^{-}=\{q \in \Omega \mid \lambda(q)<0\}$.

Next we consider complete Einstein manifolds $(M, g)$ that admit a non-constant solution $\lambda$ to (2.1).

Theorem 2.2. Let $\left(M^{n}, g\right)$ be a connected, complete manifold without boundary. Suppose $g$ is Einstein with $\operatorname{Ric}(g)=(n-1) \kappa g$, where $\kappa=0,1$ or -1 . Suppose there exists a non-constant solution $\lambda$ to the equation

$$
-\left(\Delta_{g} \lambda\right) g+\nabla_{g}^{2} \lambda-\lambda \operatorname{Ric}(g)=g .
$$

(i) If $\kappa=1$, then $\left(M^{n}, g\right)$ is isometric to $\mathbb{S}^{n}$.

(ii) If $\kappa=0$, then $\left(M^{n}, g\right)$ is isometric to $\mathbb{R}^{n}$.

(iii) If $\kappa=-1$, then $\left(M^{n}, g\right)$ is isometric to $\mathbb{H}^{n}$ provided $\nabla \lambda(p)=0$ for some $p$. If $\nabla \lambda \neq 0$ everywhere, then $(M, g)$ is isometric to $\left(\mathbb{R}^{1} \times \Sigma, d s^{2}+\cosh ^{2} s g_{0}\right)$ and $\lambda$ is given by $A \sinh s+\frac{1}{n-1}$ for some constant $A>0$. Here $\left(\Sigma, g_{0}\right)$ is a complete Einstein manifold satisfying $\operatorname{Ric}\left(g_{0}\right)=-(n-2) g_{0}$. In particular, $\left(M^{n}, g\right)$ has constant sectional curvature -1 if $n \leq 4$.

Proof. (i) If $\kappa=1$, then $M$ is compact with diameter $d \leq \pi$. Choose $p \in M$ such that $\nabla \lambda(p)=0$. Let $\alpha(s)$ be a geodesic defined on $[0, \infty)$ with $\alpha(0)=p$. By (i) in Lemma 2.1. $\lambda(\alpha(\pi)) \neq \lambda(p)$; hence $\alpha(\pi) \neq p$. By (ii) in Lemma 2.1 $\alpha(s)$ is minimizing on $[0, \pi]$. Hence, $d \geq \pi$. Therefore $(M, g)$ is isometric to $\mathbb{S}^{n}$ by the maximal diameter theorem [3].

(ii) Suppose $\kappa=0$; we show that $\lambda$ must have an absolute maximum. Let $q \in M$ be any given point. The exponential map $\exp _{q}(\cdot): T_{q} M \rightarrow M$ is surjective, where $T_{q} M$ is the tangent space of $M$ at $q$. Define $\tilde{\lambda}=\lambda \circ \exp _{q}$. Let $S_{q}$ be the unit sphere in $T_{q} M$. For any $v \in S_{q}$ and any $s \geq 0$, (2.2) implies

$$
\frac{d^{2}}{d s^{2}} \tilde{\lambda}(s v)=-\frac{1}{n-1} \text {. }
$$

Since $\tilde{\lambda}(0)=\lambda(q)$ and $\frac{d}{d s} \tilde{\lambda}(s v)(0)=\langle\nabla \lambda(q), v\rangle$, (2.9) implies

$$
\tilde{\lambda}(s v)=-\frac{1}{2(n-1)} s^{2}+\langle\nabla \lambda(q), v\rangle s+\lambda(q) .
$$

Since $|\langle\nabla \lambda(q), v\rangle| \leq|\nabla \lambda(q)|$, we have $\lim _{s \rightarrow \infty} \tilde{\lambda}(s v)=-\infty$ uniformly with respect to $v \in S_{q}$. In particular, $\tilde{\lambda}$ has an absolute maximum. Therefore, $\lambda$ has an absolute maximum. Consequently, there exists $p \in M$ such that $\nabla \lambda(p)=0$. By (ii) in Lemma 2.1, the injectivity radius of $(M, g)$ at $p$ is $\infty$. Hence, we can proceed as in the proof of Theorem 2.1 to conclude that $\left(M^{n}, g\right)$ is isometric to $\mathbb{R}^{n}$.

(iii) Suppose $\kappa=-1$. If $\nabla \lambda=0$ somewhere, we can proceed as in the proof of Theorem 2.1 to conclude that $\left(M^{n}, g\right)$ is isometric to the hyperbolic space $\mathbb{H}^{n}$. In what follows, we assume that $\nabla \lambda$ is never zero. For $a \in \mathbb{R}$, let $\lambda_{a}$ be the level set $\{\lambda=a\}$. Then $\lambda_{a}$ is a smooth hypersurface whenever it is non-empty. By Lemma 2.2. $|\nabla \lambda|$ is constant on each connected component of $\lambda_{a}$.

Choose $a$ such that $\lambda_{a}$ is non-empty. Let $\Sigma$ be a connected component of $\lambda_{a}$ and let $b>0$ be the constant that equals $|\nabla \lambda|$ on $\Sigma$. Let $p \in \Sigma$ be any chosen point. Let 
$\gamma(s)$ be the geodesic defined on $(-\infty, \infty)$ such that $\gamma(0)=p$ and $\gamma^{\prime}(0)=b^{-1} \nabla \lambda(p)$. Then $f(s)=\lambda(\gamma(s))$ satisfies $f(0)=a, f^{\prime}(0)=b$ and

$$
\frac{d^{2} f}{d s^{2}}-f=-\frac{1}{n-1} \text {. }
$$

Suppose $f^{\prime}(s)=0$ for some $s>0$. Let $s_{1}>0$ be the smallest $s>0$ such that $f^{\prime}(s)=0$. For any $0<s<s_{1}$, consider the level set $\lambda_{f(s)}$. Let $s^{\prime}=\operatorname{dist}\left(p, \lambda_{f(s)}\right)$; then $s^{\prime} \leq s$. Let $\alpha(\cdot)$ be a minimizing geodesic such that $\alpha(0)=p$ and $\alpha\left(s^{\prime}\right) \in \lambda_{f(s)}$. Let $F(s)=\lambda(\alpha(s))$. Then $F$ also satisfies (2.11) with $F(0)=a$ and $F^{\prime}(0) \leq b$. If $F^{\prime}(0)<b$, by (2.11) we have $f\left(s^{\prime}\right)>F\left(s^{\prime}\right)=f(s)$. On the other hand, the facts $s^{\prime} \leq s$ and $f$ is strictly increasing on $[0, s]$ imply $f\left(s^{\prime}\right) \leq f(s)$, hence a contradiction. Therefore, $F^{\prime}(0)=b$. In this case, we have $\alpha^{\prime}(0)=\gamma^{\prime}(0)$; hence $\alpha(t)=\gamma(t)$ for all $t \in\left[0, s^{\prime}\right]$. Since $\lambda\left(\alpha\left(s^{\prime}\right)\right)=\lambda(\gamma(s))$, we conclude $s^{\prime}=s$. Consequently, $s=$ $\operatorname{dist}\left(p, \lambda_{f(s)}\right)$ and $\gamma^{\prime}(s) \perp \lambda_{f(s)}$ at $\gamma(s)$. Since $s \in\left(0, s_{1}\right)$ is arbitrary, we have $\gamma^{\prime}\left(s_{1}\right) \perp \lambda_{f\left(s_{1}\right)}$ at $\gamma\left(s_{1}\right)$. In particular, $\gamma^{\prime}\left(s_{1}\right)$ and $\nabla \lambda\left(\gamma\left(s_{1}\right)\right)$ are parallel; hence $f^{\prime}\left(s_{1}\right)=\left\langle\gamma^{\prime}\left(s_{1}\right), \nabla \lambda\left(\gamma\left(s_{1}\right)\right)\right\rangle \neq 0$. This contradicts the assumption $f^{\prime}\left(s_{1}\right)=0$. Therefore, $f^{\prime}(s) \neq 0$ for all $s>0$. Similarly, we can prove that $f^{\prime}(s) \neq 0$ for $s<0$.

Now we have $f^{\prime}(s)>0$ for all $s$. Moreover, by the above proof, we have $\gamma^{\prime}(s) \perp$ $\lambda_{f(s)}$ at $\gamma(s)$ for all $s$. Hence,

$$
\nabla(\lambda(s))=\phi(s) \gamma^{\prime}(s)
$$

for some smooth positive function $\phi(s)$ defined on $(-\infty, \infty)$. Therefore, after reparametrization, $\gamma$ is an integral curve of the vector field $\nabla \lambda$. In particular, two different $\gamma$ will not intersect. Since any point in $M$ lies on a geodesic that is perpendicular to $\Sigma$, we conclude that $(M, g)$ is isometric to $\left(\mathbb{R}^{1} \times \Sigma, d s^{2}+g_{s}\right)$, where $\{s\} \times \Sigma$ is the level set of $\operatorname{dist}(\cdot, \Sigma)$ and $g_{s}$ is the induced metric on $\{s\} \times \Sigma$. Moreover, by (2.11) and the fact $\lambda$ and $|\nabla \lambda|$ are constants on $\Sigma$, we know $\lambda$ depends only on $s$ and $\lambda=\lambda(s)$ is given by

$$
\lambda(s)=A \sinh s+B \cosh s+\frac{1}{n-1}
$$

for some constants $A$ and $B$. Since $|\nabla \lambda|=\left|\lambda^{\prime}\right|$, which is never zero, by reversing $\frac{\partial}{\partial s}$, we may assume that $\lambda^{\prime}(s)>0$ for all $s$. Let $A_{s}$ be the second fundamental form of $\{s\} \times \Sigma$ w.r.t. $\frac{\partial}{\partial s}$. By Lemma 2.2 and (2.13), we have

$$
\frac{\partial}{\partial s} g_{s}=2 A_{s}=2|\nabla \lambda|^{-1}\left(\lambda-\frac{1}{n-1}\right) g_{s}=2 \frac{\lambda^{\prime \prime}}{\lambda^{\prime}} g_{s} .
$$

Therefore, we conclude $g_{s}=\phi^{2}(s) g_{0}$, where

$$
\phi(s)=\frac{\lambda^{\prime}(s)}{\lambda^{\prime}(0)}=A^{-1}(A \cosh s+B \sinh s) .
$$

Since $\lambda^{\prime}>0$, we have $A>0$ and $A \geq|B|$. If $A=|B|$, then $\phi(s)=e^{s}$ or $e^{-s}$, and the metric $g$ is not complete. Hence, $A>|B|$. Therefore, $\lambda=\frac{1}{n-1}$ somewhere. By translating $s$, we may assume $\lambda(0)=\frac{1}{n-1}$. Then $\lambda(s)=A \sinh s+\frac{1}{n-1}, \phi(s)=$ $\cosh s$, and

$$
g=d s^{2}+\cosh ^{2} s g_{0} .
$$

Using the fact $\operatorname{Ric}(g)=-(n-1) g$ and (3.3) in Lemma 3.1 in the next section, we have $\operatorname{Ric}\left(g_{0}\right)=-(n-2) g_{0}$. When $n=4$, this implies $g_{0}$ has constant sectional curvature -1 ; hence $g$ has constant sectional curvature -1 by (2.15). 
Let $\left(\Sigma, g_{0}\right)$ be any complete Einstein manifold with negative scalar curvature which is not a space form. Suppose $\operatorname{Ric}\left(g_{0}\right)=-(n-1) g_{0}$. Consider the warped product $(M, g)=\left(\mathbb{R}^{1} \times \Sigma, d s^{2}+\cosh ^{2} s g_{0}\right)$. Define $\lambda=A \sinh s+\frac{1}{n-1}$ on $M$, where $A>0$ is a constant. It is easy to verify that $\lambda$ is a solution to (2.8). In this case, $(M, g)$ is complete, Einstein, but is not a space form.

\section{WARPED-PRODUCT CRITICAL METRICS}

In this section, we first seek a general procedure to construct warped-product metrics $g$ which satisfy

$$
-\left(\Delta_{g} \lambda\right) g+\nabla_{g}^{2} \lambda-\lambda \operatorname{Ric}(g)=g
$$

for some function $\lambda$. Then we construct examples of critical metrics with disconnected boundary and non-Einstein critical metrics whose boundary is a standard round sphere. The first part of our discussion is motivated by the work of Kobayashi in 8 .

Let $(N, h)$ be a Riemannian manifold of dimension $n-1$. Let $I \subset \mathbb{R}^{1}$ be an open interval and $d s^{2}$ be the standard metric on $I$. Let $r$ be a smooth positive function on $I$. Consider the warped-product metric

$$
g=d s^{2}+r^{2} h
$$

on $M=I \times N$.

Lemma 3.1. $\quad$ (i) The Ricci curvature of $g$ is given by

$$
\begin{gathered}
\operatorname{Ric}(g)\left(\partial_{s}, \partial_{s}\right)=-(n-1) \frac{r^{\prime \prime}}{r}, \\
\left.\operatorname{Ric}(g)\right|_{T N}=\operatorname{Ric}(h)-\left.\left[(n-2)\left(\frac{r^{\prime}}{r}\right)^{2}+\frac{r^{\prime \prime}}{r}\right] g\right|_{T N}, \\
\operatorname{Ric}\left(\partial_{s}, X\right)=0, \forall X \in T N,
\end{gathered}
$$

where " I" denotes the derivative taken with respect to $s \in I, \operatorname{Ric}(h)$ is the Ricci curvature of $h$ and $T N$ denotes the tangent space to $N$. Consequently,

$$
R(g)=-2(n-1)\left(\frac{r^{\prime \prime}}{r}\right)+\frac{R(h)}{r^{2}}-(n-1)(n-2)\left(\frac{r^{\prime}}{r}\right)^{2},
$$

where $R(g), R(h)$ are the scalar curvature of $g, h$, respectively.

(ii) Suppose $\lambda$ is a smooth function on $M$ depending only on $s$. Then

$$
\nabla_{g}^{2} \lambda\left(\partial_{s}, \partial_{s}\right)=\lambda^{\prime \prime},\left.\nabla_{g}^{2} \lambda\right|_{T N}=\left.\left(\frac{r^{\prime}}{r}\right) \lambda^{\prime} g\right|_{T N}, \nabla_{g}^{2} \lambda\left(\partial_{s}, X\right)=0
$$

where $X \in T N$.

Proof. (i) is standard; see [2]. Direct computations give (ii).

To proceed, we note that (3.1) implies

$$
\Delta_{g} \lambda=-\frac{1}{n-1}(R \lambda+n)
$$

hence, (3.1) is equivalent to

$$
\nabla_{g}^{2} \lambda=\lambda \text { Ric }-\frac{R \lambda+1}{n-1} g .
$$


Proposition 3.1. For any constant $R$, the metric $g$ has constant scalar curvature $R$ and satisfies (3.1) for a smooth function $\lambda$ depending only on $s \in I$, if and only if the following holds:

(i) $(N, h)$ is an Einstein manifold with $\operatorname{Ric}(h)=(n-2) \kappa_{0} h$, the function $r$ satisfies

$$
r^{\prime \prime}+\frac{R}{n(n-1)} r=a r^{1-n}
$$

for some constant $a$, and the constant $\kappa_{0}$ satisfies

$$
\left(r^{\prime}\right)^{2}+\frac{R}{n(n-1)} r^{2}+\frac{2 a}{n-2} r^{2-n}=\kappa_{0} .
$$

(ii) The function $\lambda$ satisfies

$$
r^{\prime} \lambda^{\prime}-r^{\prime \prime} \lambda=-\frac{1}{n-1} r .
$$

Proof. Suppose $g$ has constant scalar curvature $R$ and there is a smooth function $\lambda=\lambda(s)$ satisfying (3.1). Since $\lambda$ cannot be identically zero, there exists $s_{0} \in I$ such that $\lambda\left(s_{0}\right) \neq 0$. At $s_{0}$, by Lemma 3.1 and (3.8), we have

$$
\begin{aligned}
\operatorname{Ric}(h) & =\left.\operatorname{Ric}(g)\right|_{T N}+\left.\left[(n-2)\left(\frac{r^{\prime}}{r}\right)^{2}+\frac{r^{\prime \prime}}{r}\right] g\right|_{T N} \\
& =\left.\frac{1}{\lambda}\left(\nabla_{g}^{2} \lambda+\frac{R \lambda+1}{n-1} g\right)\right|_{T N}+\left.\left[(n-2)\left(\frac{r^{\prime}}{r}\right)^{2}+\frac{r^{\prime \prime}}{r}\right] g\right|_{T N} \\
& =\left.\left[\frac{1}{\lambda}\left(\frac{r^{\prime} \lambda^{\prime}}{r}+\frac{R \lambda+1}{n-1}\right)+(n-2)\left(\frac{r^{\prime}}{r}\right)^{2}+\frac{r^{\prime \prime}}{r}\right] g\right|_{T N} .
\end{aligned}
$$

Since $R$ is a constant and $r$ and $\lambda$ depend only on $s$, (3.12) implies that $(N, h)$ is Einstein. Suppose $\operatorname{Ric}(h)=(n-2) \kappa_{0} h$, where $\kappa_{0}$ is a constant.

Evaluating both sides of (3.1) at $\partial_{s}$, using Lemma 3.1 and the fact that

$$
\nabla^{2} \lambda\left(\partial_{s}, \partial_{s}\right)-\Delta_{g} \lambda=-(n-1) \frac{r^{\prime}}{r} \lambda^{\prime}
$$

we have

$$
-(n-1) \frac{r^{\prime}}{r} \lambda^{\prime}+(n-1) \frac{r^{\prime \prime}}{r} \lambda=1,
$$

which proves (ii).

Differentiating (3.11), using (3.7), (3.11) and the fact that

$$
\Delta_{g} \lambda=\lambda^{\prime \prime}+(n-1) \frac{r^{\prime} \lambda^{\prime}}{r}
$$

we have

$$
\begin{aligned}
-\frac{r^{\prime}}{n-1} & =r^{\prime} \lambda^{\prime \prime}-r^{\prime \prime \prime} \lambda \\
& =\left(\Delta_{g} \lambda-(n-1) \frac{r^{\prime} \lambda^{\prime}}{r}\right) r^{\prime}-r^{\prime \prime \prime} \lambda \\
& =\left(-\frac{R \lambda+n}{n-1}-(n-1) \frac{r^{\prime \prime} \lambda}{r}+1\right) r^{\prime}-r^{\prime \prime \prime} \lambda .
\end{aligned}
$$


Hence

$$
\left[r^{\prime \prime \prime}+(n-1) \frac{r^{\prime} r^{\prime \prime}}{r}+\frac{R}{n-1} r^{\prime}\right] \lambda=0 .
$$

By (3.11), if $\lambda(s)=0$, then $\lambda^{\prime}(s) \neq 0$. Hence the set $\{s \in I \mid \lambda \neq 0\}$ is dense in $I$. So (3.13) shows

$$
r^{\prime \prime \prime}+(n-1) \frac{r^{\prime} r^{\prime \prime}}{r}+\frac{R}{n-1} r^{\prime} \equiv 0
$$

in $I$. Multiplying (3.14) by $r^{n-1}$ and using the fact that $R$ is a constant and $r>0$, we conclude from (3.14) that

$$
\left[r^{n-1} r^{\prime \prime}+\frac{R}{n(n-1)} r^{n}\right]^{\prime}=0
$$

which is equivalent to

$$
r^{\prime \prime}+\frac{R}{n(n-1)} r=a r^{1-n}
$$

for some constant $a$. Now (3.10) follows directly from (3.9), (3.5) and the fact $R(h)=(n-1)(n-2) \kappa_{0}$.

Conversely, suppose $(N, h)$ is Einstein with $\operatorname{Ric}(h)=(n-2) \kappa_{0} h$ and the functions $r, \lambda$ satisfy (3.9)-(3.11). Let $g=d s^{2}+r^{2} h$. By Lemma 3.1 the scalar curvature $R(g)$ of $g$ is given by

$$
R(g)=-2(n-1)\left(\frac{r^{\prime \prime}}{r}\right)+\frac{(n-1)(n-2) \kappa_{0}}{r^{2}}-(n-1)(n-2)\left(\frac{r^{\prime}}{r}\right)^{2} .
$$

Hence, $R(g)=R$ by (3.9) and (3.10). Next, suppose $X, Y \in T N$. By Lemma 3.1 and (3.9)-(3.11), we have

$$
\begin{aligned}
& \lambda \operatorname{Ric}(g)(X, Y)-\frac{R \lambda+1}{n-1} g(X, Y) \\
= & {\left[\frac{(n-2) \lambda \kappa_{0}}{r^{2}}-(n-2) \lambda\left(\frac{r^{\prime}}{r}\right)^{2}-\frac{r^{\prime \prime} \lambda}{r}-\frac{R \lambda+1}{n-1}\right] g(X, Y) } \\
= & \frac{r^{\prime} \lambda^{\prime}}{r} g(X, Y)=\nabla_{g}^{2} \lambda(X, Y)
\end{aligned}
$$

and

$$
\nabla_{g}^{2} \lambda\left(\partial_{s}, X\right)=0=\lambda \operatorname{Ric}(g)\left(\partial_{s}, X\right)-\frac{R \lambda+1}{n-1} g\left(\partial_{s}, X\right) .
$$

On the other hand, differentiating (3.9), (3.11) and canceling $r^{\prime \prime \prime}$, we have

$$
r^{\prime} \lambda^{\prime \prime}+\left[(n-1) a r^{-n}+\frac{R}{n(n-1)}\right] r^{\prime} \lambda=-\frac{r^{\prime}}{n-1} \text {. }
$$

By (3.11), if $r^{\prime}(s)=0$, then $r^{\prime \prime}(s) \neq 0$. Hence the set $\left\{r^{\prime}(s) \in I \mid \lambda \neq 0\right\}$ is dense in I. So (3.18) implies

$$
\lambda^{\prime \prime}+\left[(n-1) a r^{-n}+\frac{R}{n(n-1)}\right] \lambda=-\frac{1}{n-1} .
$$

By (3.9), (3.19) becomes

$$
\lambda^{\prime \prime}+\left[(n-1) \frac{r^{\prime \prime}}{r}+\frac{R}{n-1}\right] \lambda=-\frac{1}{n-1},
$$


from which we see that

$$
\nabla_{g}^{2} \lambda\left(\partial_{s}, \partial_{s}\right)=\lambda \operatorname{Ric}(g)\left(\partial_{s}, \partial_{s}\right)-\frac{R \lambda+1}{n-1} g\left(\partial_{s}, \partial_{s}\right),
$$

by Lemma 3.1

By (3.16), (3.17) and (3.21), we conclude that $\lambda$ satisfies (3.1). This completes the proof of the proposition.

Remark 3.1. The constant $a$ in (3.9) has a geometric interpretation. Assuming $r$ and $(N, h)$ satisfy (i) and (ii) in Proposition 3.1 then it follows from Lemma 3.1 and (3.9) that

$$
\begin{aligned}
\operatorname{Ric}(g)\left(\partial_{s}, \partial_{s}\right) & =-(n-1) a r^{-n}+\frac{R}{n}, \\
\left.\operatorname{Ric}(g)\right|_{T N} & =\left.\left(a r^{-n}+\frac{R}{n}\right) g\right|_{T N} .
\end{aligned}
$$

Hence, $a=0$ if and only if $g$ is an Einstein metric.

Remark 3.2. The condition (3.9) on the function $r$ in Proposition 3.1 turns out to be the same condition that Kobayashi obtained in 8, where he constructed warped-product solutions to an equation, similar to (3.1),

$$
-\left(\Delta_{g} f\right) g+\nabla_{g}^{2} f-f \operatorname{Ric}(g)=0,
$$

where the metric $g$ and the function $f$ are the unknowns. Kobayashi proved that, if $(N, h)$ has constant sectional curvature, then $g=d s^{2}+r^{2} h$ satisfies (3.23) with some function $f=f(s)$ if and only if (3.9) holds (see Lemma 1.1 in [8]). Equation (3.23) is interesting to study because of its root in general relativity (see [5, 9], [4, etc.).

Next, we consider the function $\lambda$ in Proposition 3.1. Viewed as an ODE about $\lambda$, equation (3.11) becomes singular at points where $r^{\prime}$ is zero. Nonetheless, we show that it always has a solution $\lambda$ as long as $r$ is a non-constant solution to (3.9).

Lemma 3.2. Suppose $r$ is a smooth, positive, non-constant solution to

$$
r^{\prime \prime}+\frac{R}{n(n-1)} r=a r^{1-n}
$$

on $I$, where $R$ and a are some given constants. Then

(i) $r^{\prime}$ and $r^{\prime \prime}$ cannot vanish simultaneously at any point in $I$.

(ii) Suppose $r^{\prime}\left(s_{0}\right) \neq 0, s_{0} \in I$. Given any initial condition $\lambda\left(s_{0}\right)=c$, there is a unique solution $\lambda$ of (3.11) on I such that $\lambda\left(s_{0}\right)=c$.

(iii) Suppose $r^{\prime \prime}\left(s_{0}\right) \neq 0, s_{0} \in I$. Given any initial condition $\lambda^{\prime}\left(s_{0}\right)=c$, there is a unique solution $\lambda$ of (3.11) on I such that $\lambda^{\prime}\left(s_{0}\right)=c$.

(iv) Any two solutions to (3.11) differ by a constant multiple of $r^{\prime}$.

Proof. (i) Taking the derivative of (3.24),

$$
r^{\prime \prime \prime}+\left[\frac{R}{n(n-1)}+(n-1) a r^{-n}\right] r^{\prime}=0 .
$$

Suppose $r^{\prime}\left(s_{0}\right)=r^{\prime \prime}\left(s_{0}\right)=0$ for some $s_{0} \in I$; then $r^{\prime} \equiv 0$ by the uniqueness of solutions to the ODE (3.25). Since $r$ is non-constant, this is impossible. 
(ii) Suppose $r^{\prime}\left(s_{0}\right) \neq 0$ and $c$ is given. On $I$, we can solve for $\lambda$

$$
\lambda^{\prime \prime}+\left[\frac{R}{n(n-1)}+(n-1) a r^{-n}\right] \lambda=-\frac{1}{n-1}
$$

with initial data $\lambda\left(s_{0}\right)=c$ and $\lambda^{\prime}\left(s_{0}\right)=\frac{1}{r^{\prime}\left(s_{0}\right)}\left[c r^{\prime \prime}\left(s_{0}\right)-\frac{r\left(s_{0}\right)}{n-1}\right]$. Let $\lambda$ be such a solution to (3.26). By (3.25) and (3.26), we have

$$
\left(r^{\prime} \lambda^{\prime}-r^{\prime \prime} \lambda+\frac{r}{n-1}\right)^{\prime}=0
$$

on $I$. Since $r^{\prime} \lambda^{\prime}-r^{\prime \prime} \lambda+\frac{r}{n-1}=0$ at $s_{0}, \lambda$ satisfies (3.11) with $\lambda\left(s_{0}\right)=c$.

Conversely, if $\lambda$ is a solution of (3.11) with $\lambda\left(s_{0}\right)=c$, we must have $\lambda^{\prime}\left(s_{0}\right)=$ $\frac{1}{r^{\prime}\left(s_{0}\right)}\left[c r^{\prime \prime}\left(s_{0}\right)-\frac{r\left(s_{0}\right)}{n-1}\right]$ since $r^{\prime}\left(s_{0}\right) \neq 0$. On the other hand, $\lambda$ satisfies (3.26) by the proof of Proposition 3.1. Hence, $\lambda$ is unique.

(iii) can be proved in the same way as (ii) is proved.

(iv) Let $\lambda_{1}, \lambda_{2}$ be any two solutions to (3.11) on $I$. Let $\phi=\lambda_{1}-\lambda_{2}$. Then $\phi$ satisfies $r^{\prime} \phi^{\prime}-r^{\prime \prime} \phi=0$, which implies $\phi$ is a constant multiple of $r^{\prime}$ on any subinterval of $I$ where $r^{\prime}$ is never zero. By (i), the roots of $r^{\prime}$ are isolated in $I$ and $r^{\prime}(s)=0$ implies $r^{\prime \prime}(s) \neq 0$. Therefore, $\phi=C r^{\prime}$ on $I$ for some constant $C$.

In what follows, we always assume $R$ and $a$ are two given constants. By Proposition 3.1 and Lemma 3.2. any non-constant, positive solution $r$ to the ODE

$$
r^{\prime \prime}+\frac{R}{n(n-1)} r=a r^{1-n},
$$

on an interval $I$, will give rise to a metric $g=d s^{2}+r^{2} h$, on $M=I \times N$, which satisfies (3.1) for some function $\lambda$ (provided $(N, h)$ is an Einstein manifold with Ricci curvature properly chosen). It is natural to know if one can obtain a compact $(M, g)$ from this procedure such that $\lambda=0$ on $\partial M$. For this purpose, we consider solutions $r$ to (3.27) existing on $\mathbb{R}^{1}$ and ask how many roots the associated solutions $\lambda$ to (3.11) may have.

The following lemma was proved by Kobayashi in 8 .

Lemma 3.3. Suppose $a>0$. Then any local positive solution to (3.27) can be extended as a positive solution on $\mathbb{R}^{1}$. If in addition $R>0$, then each non-constant solution on $\mathbb{R}^{1}$ is periodic.

For reasons which will be clear in Lemma 4.3, we impose the assumption $a>0$ hereafter. For any positive solution $r$ to (3.27) on $\mathbb{R}^{1}$, there exists a constant $\kappa_{0}$ such that

$$
\left(r^{\prime}\right)^{2}+\frac{R}{n(n-1)} r^{2}+\frac{2 a}{n-2} r^{2-n}=\kappa_{0} .
$$

As $a>0$, it follows directly from (3.28) that $r$ is bounded from below by a positive constant.

Lemma 3.4. Suppose $a>0$. Let $r$ be a non-constant, positive solution to (3.27) on $\mathbb{R}^{1}$. If $R \leq 0$, then $r^{\prime}(s)$ has a unique root. If $R>0$, then $r^{\prime}(s)=0$ if and only if $r(s)$ is the maximum or the minimum of $r$. 
Proof. Suppose $R \leq 0$; then (3.27) implies $r^{\prime \prime} \geq a r^{1-n}$. Assume $r^{\prime}>0$ everywhere; then $r(s) \leq r(0)$ for all $s \leq 0$. So $r^{\prime \prime}(s) \geq C$ for some positive constant $C$ for $s<0$. This implies $r^{\prime}(s)<0$ somewhere, which is a contradiction. Similarly, it is impossible to have $r^{\prime}<0$ everywhere. Hence $r^{\prime}(s)=0$ for some $s$. Since $r^{\prime \prime}>0$, the root of $r^{\prime}(s)$ is unique.

Suppose $R>0$; then $r$ is periodic by Lemma 3.3. Let $r_{\max }$ and $r_{\min }$ be the maximum and minimum of $r$. If $r^{\prime}\left(s_{0}\right)=0$, then 3.28) implies

$$
\frac{R}{n(n-1)} r^{2}\left(s_{0}\right)+\frac{2 a}{n-2} r^{2-n}\left(s_{0}\right)=\kappa_{0}
$$

with $\kappa_{0}>0$. In particular, (3.29) holds with $r\left(s_{0}\right)$ replaced by $r_{\max }$ or $r_{\min }$. Consider

$$
F(r)=\frac{R}{n(n-1)} r^{2}+\frac{2 a}{n-2} r^{2-n}
$$

as a function of $r$. Then

$$
\frac{d F}{d r}=2 r\left[\frac{R}{n(n-1)}-a r^{-n}\right] .
$$

Let $r_{0}=\left(\frac{n(n-1) a}{R}\right)^{\frac{1}{n}}$. Then $F(r)$ is strictly decreasing on $\left(0, r_{0}\right)$ and strictly increasing on $\left(r_{0}, \infty\right)$. So for $\kappa_{0}>0, F(r)=\kappa_{0}$ has at most 2 distinct solutions. Hence, $r\left(s_{0}\right)$ is one of $r_{\min }$ and $r_{\max }$. Moreover, as $r$ is assumed not to be a constant, we have

$$
r_{\min }<r_{0}<r_{\max }
$$

Let $r$ be given as in Lemma [3.4] Without losing generality, we may assume $r^{\prime}(0)=0$. By the uniqueness of solutions of ODEs, $r$ is an even function. In case $R>0$ and $r$ is non-constant, the roots of $r^{\prime}(s)$ form a discrete subset in $\mathbb{R}^{1}$. If we arrange it so that $r(0)=r_{\min }$ ( or $r_{\max }$ ) and if $0, \pm s_{1}, \pm s_{2}, \ldots$ are zeros of $r^{\prime}$ with $s_{1}<s_{2}<\ldots$, then $r\left( \pm s_{1}\right)=r_{\max }$ (or $r_{\min }$ respectively) and $r$ is periodic with period $s_{2}$. Now let $\lambda_{0}$ be the solution of (3.11) on $\mathbb{R}^{1}$ with $\lambda_{0}^{\prime}(0)=0$, which exists and is unique by Lemma 3.2 , then $\lambda_{0}$ is also an even function.

Proposition 3.2. Let $a>0$ and $R$ be two constants. Let $r$ be a positive, nonconstant solution to (3.27) on $\mathbb{R}^{1}$ satisfying $r^{\prime}(0)=0$. For such a given $r$, let $\lambda_{0}$ be the solution to (3.11) on $\mathbb{R}^{1}$ satisfying $\lambda_{0}^{\prime}(0)=0$. Let $\lambda$ be another solution to (3.11) on $\mathbb{R}^{1}$. By Lemma 3.2, $\lambda=\lambda_{0}+C r^{\prime}$ for some constant $C$.

(i) Suppose $R=0$. Then $\lambda(0)>0, \int_{1}^{+\infty} \frac{r}{\left(r^{\prime}\right)^{2}} d \tau=+\infty, \lambda$ has a unique positive root $\zeta_{1}$ and a unique negative root $\zeta_{2}$ and they are related by

$$
\int_{\theta}^{\zeta_{1}} \frac{r}{\left(r^{\prime}\right)^{2}} d \tau=\int_{-\theta}^{\zeta_{2}} \frac{r}{\left(r^{\prime}\right)^{2}} d \tau
$$

where $\theta$ and $-\theta$ are the unique positive and negative roots of $\lambda_{0}$.

(ii) Suppose $R<0$. Then $\lambda(0)>0, \int_{1}^{+\infty} \frac{r}{\left(r^{\prime}\right)^{2}} d \tau<+\infty, \lambda_{0}$ has a unique positive root $\theta$ and a unique negative root $-\theta$. Moreover, (a) if $C \leq$ $-\frac{1}{n-1} \int_{\theta}^{+\infty} \frac{r}{\left(r^{\prime}\right)^{2}} d \tau$, then $\lambda$ has a unique root and the root is positive; (b) if $C \geq \frac{1}{n-1} \int_{\theta}^{+\infty} \frac{r}{\left(r^{\prime}\right)^{2}} d \tau$, then $\lambda$ has a unique root and the root is negative; (c) 
if $|C|<\frac{1}{n-1} \int_{\theta}^{+\infty} \frac{r}{\left(r^{\prime}\right)^{2}} d \tau$, then $\lambda$ has a unique positive root $\zeta_{1}$ and a unique negative root $\zeta_{2}$ and $\zeta_{1}, \zeta_{2}$ are related by (3.33). In particular, $\zeta_{1}>\zeta$ and $\zeta_{2}<-\zeta$, where $\zeta \in(0, \theta)$ is the constant determined by

$$
\int_{\zeta}^{\theta} \frac{r}{\left(r^{\prime}\right)^{2}} d \tau=\int_{\theta}^{+\infty} \frac{r}{\left(r^{\prime}\right)^{2}} d \tau
$$

(iii) Suppose $R>0$. Then $\lambda$ has exactly one root between any two consecutive roots of $r^{\prime}$. If $r(0)=r_{\min }$ (respectively $r_{\max }$ ), then $\lambda(0)>0$ (respectively $<0)$. Let $\theta>0$ be the first positive root of $\lambda_{0}$. Then the smallest positive root $\zeta_{1}$ and the largest negative root of $\zeta_{2}$ of $\lambda$ are related by (3.33).

Proof. Since $r^{\prime}(0)=0$, by (3.11) we have $r^{\prime \prime}(0) \lambda(0)=\frac{r(0)}{n-1}$. In particular, $\lambda(0)$ and $r^{\prime \prime}(0)$ have the same sign.

(i) Suppose $R=0$. We have $r^{\prime \prime}=a r^{1-n}>0$ for all $s$. Hence, $\lambda(0)>0$. On $(0,+\infty)$, the function

$$
-\frac{r^{\prime}}{n-1} \int_{1}^{s} \frac{r}{\left(r^{\prime}\right)^{2}} d \tau
$$

is a solution to (3.11). By Lemma 3.2, we have

$$
\lambda(s)=r^{\prime}(s)\left(C_{1}-\frac{1}{n-1} \int_{1}^{s} \frac{r}{\left(r^{\prime}\right)^{2}} d \tau\right)
$$

for some constant $C_{1}$ for any $s>0$. Let $\kappa_{0}>0$ be the constant in (3.28) with $R=0$. Then $\left(r^{\prime}\right)^{2}<\kappa_{0}$ and $r(s) \geq r(0)>0$. Hence,

$$
\lim _{s \rightarrow+\infty} \int_{1}^{s} \frac{r}{\left(r^{\prime}\right)^{2}} d s=+\infty
$$

Since $r^{\prime}(0)=0$ and $r(0)>0$, we also have

$$
\lim _{s \rightarrow 0} \int_{1}^{s} \frac{r}{\left(r^{\prime}\right)^{2}} d s=-\infty .
$$

By (3.35)-(3.37), we conclude that $\lambda$ has a unique positive root $\zeta_{1}$. Similarly, we can prove that $\lambda$ has a unique negative root $\zeta_{2}$.

Let $\theta>0$ be the unique positive root of $\lambda_{0}$. Then $-\theta$ is its negative root because $\lambda_{0}$ is an even function. Moreover, (3.11) implies

$$
\lambda_{0}(s)= \begin{cases}-\frac{r^{\prime}(s)}{n-1} \int_{\theta}^{s} \frac{r}{\left(r^{\prime}\right)^{2}} d \tau, & \text { for } s>0, \\ -\frac{r^{\prime}(s)}{n-1} \int_{-\theta}^{s} \frac{r}{\left(r^{\prime}\right)^{2}} d \tau, & \text { for } s<0 .\end{cases}
$$

Therefore,

$$
\lambda(s)= \begin{cases}r^{\prime}(s)\left(C-\frac{1}{n-1} \int_{\theta}^{s} \frac{r}{\left(r^{\prime}\right)^{2}} d \tau\right), & \text { for } s>0, \\ r^{\prime}(s)\left(C-\frac{1}{n-1} \int_{-\theta}^{s} \frac{r}{\left(r^{\prime}\right)^{2}} d \tau\right), & \text { for } s<0 .\end{cases}
$$

Since $\lambda\left(\zeta_{1}\right)=\lambda\left(\zeta_{2}\right)=0$, (3.33) follows from (3.39).

(ii) Suppose $R<0$. Using the fact $r(s) \geq r(0)>0$, we have $r^{\prime \prime}=a r^{1-n}-$ $\frac{R}{n(n-1)} r \geq \alpha>0$ for some constant $\alpha$. In particular, this implies $\lambda(0)>0$, and $r(s) \geq \beta s^{2}$ for some $\beta>0$ for all $s>0$ sufficiently large. By (3.28),$r^{2} /\left(r^{\prime}\right)^{2}$ is bounded. Hence,

$$
\int_{1}^{+\infty} \frac{r}{\left(r^{\prime}\right)^{2}} d \tau<+\infty
$$


Similar to the proof in (i), we know there exists a constant $C_{0}$ such that

$$
\lambda_{0}(s)=r^{\prime}(s)\left(-C_{0}+\frac{1}{n-1} \int_{s}^{+\infty} \frac{r}{\left(r^{\prime}\right)^{2}} d \tau\right)
$$

for $s>0$. By the L'Hôpital rule, (3.27) and the facts $\lim _{s \rightarrow+\infty} r^{\prime}(s)=+\infty$ and $\lim _{s \rightarrow+\infty} r(s)=+\infty$, we have

$$
\lim _{s \rightarrow+\infty} \frac{r^{\prime}(s)}{n-1} \int_{s}^{+\infty} \frac{r}{\left(r^{\prime}\right)^{2}} d s=\frac{1}{n-1} \lim _{s \rightarrow+\infty} \frac{r(s)}{r^{\prime \prime}(s)}=\frac{n}{-R}
$$

On the other hand,

$$
\lambda_{0}(0)=\frac{1}{n-1} \frac{r(0)}{r^{\prime \prime}(0)}=\frac{1}{(n-1) a r^{-n}-\frac{R}{n}}<\frac{n}{-R} .
$$

Suppose $C_{0} \leq 0$. Then it follows from (3.40)-(3.42) and the fact $\lambda_{0}$ is even that $\lambda_{0}+n / R$ has an interior negative minimum. This is impossible because, by the proof in Proposition 3.1, $\lambda_{0}$ satisfies (3.19) or equivalently $\lambda_{0}+\frac{n}{R}$ satisfies

$$
\left(\lambda_{0}+\frac{n}{R}\right)^{\prime \prime}+\frac{R}{n(n-1)}\left(\lambda_{0}+\frac{n}{R}\right)=-\lambda(n-1) a r^{-n} \leq 0 .
$$

Therefore $C_{0}>0$. In particular, $\lim _{s \rightarrow+\infty} \lambda_{0}(s)=-\infty$. Since $\lambda_{0}(0)>0$ and $\lambda_{0}$ is even, we conclude from (3.40) that $\lambda_{0}$ has a unique positive root $\theta$ and a unique negative root $-\theta$. Moreover, $\theta$ and $C_{0}$ are related by

$$
C_{0}=\frac{1}{n-1} \int_{\theta}^{+\infty} \frac{r}{\left(r^{\prime}\right)^{2}} d \tau
$$

Now let $\lambda=\lambda_{0}+C r^{\prime}$ be another solution. Then

$$
\lambda(s)= \begin{cases}r^{\prime}(s)\left(C-C_{0}+\frac{1}{n-1} \int_{s}^{\infty} \frac{r}{\left(r^{\prime}\right)^{2}} d \tau\right), & \text { for } s>0 \\ r^{\prime}(s)\left(C+C_{0}-\frac{1}{n-1} \int_{-\infty}^{s} \frac{r}{\left(r^{\prime}\right)^{2}} d \tau\right), & \text { for } s<0 .\end{cases}
$$

It follows from (3.43) that (a) if $C \leq-C_{0}, \lambda$ has a unique root and the root is positive; (b) if $C \geq C_{0}, \lambda$ has a unique root and the root is negative; (c) if $|C|<C_{0}, \lambda$ has a unique positive root $\zeta_{1}$ and a unique negative root $\zeta_{2}$ and $\zeta_{1}, \zeta_{2}$ satisfy (3.33); moreover, (3.33) implies that

$$
\int_{\theta}^{\zeta_{1}} \frac{r}{\left(r^{\prime}\right)^{2}} d \tau>\int_{-\theta}^{-\infty} \frac{r}{\left(r^{\prime}\right)^{2}} d \tau=-\int_{\theta}^{\infty} \frac{r}{\left(r^{\prime}\right)^{2}} d \tau=\int_{\theta}^{\zeta} \frac{r}{\left(r^{\prime}\right)^{2}} d \tau .
$$

Therefore, $\zeta_{1}>\zeta$. Similarly, we have $\zeta_{2}<-\zeta$.

(iii) Suppose $R>0$. Let $\left\{s_{k}\right\}$ be the increasing positive sequence such that $\left\{0, \pm s_{1}, \pm s_{2}, \ldots\right\}$ is the set of roots of $r^{\prime}(s)$. By (3.11), $\lambda\left(s_{k}\right)$ (or $\left.\lambda\left(-s_{k}\right)\right)$ has the same sign as $r^{\prime \prime}\left(s_{k}\right)$ (or $r^{\prime \prime}\left(-s_{k}\right)$ ). Suppose $r(0)=r_{\min }$. Then $r\left(s_{1}\right)=r_{\max }$ and $r^{\prime}>0$ in $\left(0, s_{1}\right)$. Moreover, we have $r^{\prime \prime}(0)>0$ and $r^{\prime \prime}\left(s_{1}\right)<0$, which imply $\lambda(0)>0$ and $\lambda\left(s_{1}\right)<0$. Hence, $\lambda\left(\zeta_{1}\right)=0$ for some $\zeta_{1} \in\left(0, s_{1}\right)$. By (3.11), we have

$$
\lambda(s)=-\frac{r^{\prime}(s)}{n-1} \int_{\zeta_{1}}^{s} \frac{r}{\left(r^{\prime}\right)^{2}} d \tau
$$

for any $s \in\left(0, s_{1}\right)$, which shows $\zeta_{1}$ is the unique root of $\lambda$ in $\left(0, s_{1}\right)$. Similar arguments prove that $\lambda$ has a unique root between any two consecutive roots of $r^{\prime}$. Let $\zeta_{2}$ be the maximum negative root of $\lambda$. The claim that $\zeta_{1}$ and $\zeta_{2}$ satisfy (3.33) 
follows from the same proof as in (i) and (ii). The case $r(0)=r_{\max }$ can be proved similarly.

Now we are in a position to construct compact manifolds with boundary with a non-Einstein critical metric.

Examples. (1) Given $a>0$ and $R$ two constants, let $r$ be a positive solution to (3.9) on $\mathbb{R}^{1}$ satisfying $r^{\prime}(0)=0$. Let $\kappa_{0}$ be an integral constant of (3.9) so that (3.10) holds for $r$. Let $(N, h)$ be an $(n-1)$-dimensional, connected, closed Einstein manifold satisfying $\operatorname{Ric}(h)=(n-2) \kappa_{0} h$. We note that $\kappa_{0}$ must be positive if $R \geq 0$ and $\kappa_{0}$ can be arbitrary if $R<0$. Let $\lambda_{0}$ be the solution to (3.11) on $\mathbb{R}^{1}$ with $\lambda_{0}^{\prime}(0)=0$. Let $\theta$ and $-\theta$ be the unique positive and negative roots of $\lambda_{0}$. Let $\zeta_{1}>0$ and $\zeta_{2}<0$ be chosen such that (3.33) holds. Define $I=\left[\zeta_{2}, \zeta_{1}\right]$. Then $(\Omega, g)=\left(I \times N, d s^{2}+r^{2} h\right)$ satisfies (3.1) for some $\lambda$ vanishing on $\partial \Omega$. In this case, $g$ has constant scalar curvature $R$ and $\partial \Omega$ has two connected components.

(2) Let $I$ and $(\Omega, g)$ be given as in (1) with $\zeta_{1}=\theta$ and $\zeta_{2}=-\theta$. Suppose $G$ is a finite subgroup of isometries of $(N, h)$ which acts freely on $N$. Consider the action of $G \times \mathbb{Z}_{2}$ on $\Omega$ defined by

$$
(\alpha, k)(s, x)=\left((-1)^{k} s, \alpha(x)\right),
$$

where $\alpha \in G$ and $k \in \mathbb{Z}_{2}=\{0,1\}$. This is an action of isometry on $(\Omega, g)$. Suppose $H$ is a subgroup of $G \times \mathbb{Z}_{2}$ which does not contain (id, 1), where id denotes the identity map on $N$. If $(\alpha, 1) \in H$, then $(\alpha, 0) \notin H$, for otherwise (id, 1$)=$ $(\alpha, 1)\left(\alpha^{m-1}, 0\right)$ would be in $H$ (here $m$ is the order of $\alpha$ in $G$ ). From this it can be easily checked that $H$ acts freely on $\Omega$. Since $(\Omega, g)$ is compact with boundary, so is the quotient manifold $(\Omega, g) / H$. The function $\lambda_{0}$ descends to a function $\lambda$ on $(\Omega, g) / H$ which satisfies (3.1) and vanishes on the boundary $\partial(\Omega / H)$.

If $H \neq H \cap(G \times\{0\})$, we claim that $\partial(\Omega / H)$ is connected. To see this, let $\pi$ be the natural projection map from $\Omega$ to $\Omega / H$. Then

$$
\partial(\Omega / H)=\pi(\partial \Omega)=\pi(\{\theta\} \times N) \cup \pi(\{-\theta\} \times N) .
$$

Suppose $(s, x) \in \partial \Omega$, say $s=\theta$. Then $\pi(\theta, x)=\pi(-\theta, \alpha(x))$, where $(\alpha, 1)$ is an element in $H$ but not in $H \cap(G \times\{0\})$. Hence,

$$
\pi(\{\theta\} \times N) \cap \pi(\{-\theta\} \times N) \neq \emptyset,
$$

which implies $\partial(\Omega / H)$ is connected. In the special case when $(N, h)$ admits an isometry $\alpha$ without fixed points so that $\alpha^{2}=\mathbf{i d}$, we can take $G=\{\mathbf{i d}, \alpha\}$ and $H=\{(\mathbf{i d}, 0),(\alpha, 1)\}$. Then $(\Omega, g) / H$ has a connected boundary that is isometric to a constant re-scaling of $(N, h)$.

In the above construction, suppose $R \leq 0, r$ is chosen such that $\kappa_{0}=1$ and $(N, h)$ is taken to be $\mathbb{S}^{n-1}$. Then $g=d s^{2}+r^{2} h$ is simply the usual spatial Schwarzschild metric or Ads-Schwarzschild metric whose mass is given by the constant $a$. To see this, one can make a change of variable $s=s(r)$ and use (3.28) to re-write $g$ as

$$
g=\frac{1}{1-\frac{R}{n(n-1)} r^{2}-\frac{2 a}{n-2} r^{2-n}} d r^{2}+r^{2} d h .
$$

Note that the antipodal map $\alpha$ on $\mathbb{S}^{n-1}$ is an isometry without fixed points such that $\alpha^{2}=$ id. Hence, the following results follow directly from the above construction and Proposition 3.2 (i) and (ii). 
Corollary 3.1. Let $(M, g)$ be a complete, spatial Schwarzschild manifold with positive mass. Let $\Sigma_{0}$ be the horizon in $(M, g)$ (i.e. the unique closed minimal surface in $(M, g))$. The following are true:

(i) There exist functions $\lambda$ on $(M, g)$ satisfying (3.1).

(ii) Let $M_{+}, M_{-}$be the two components of $M \backslash \Sigma_{0}$. Then for any rotationally symmetric sphere $\Sigma_{\zeta_{1}}$ in $M_{+}$, there exists a rotationally symmetric sphere $\Sigma_{\zeta_{2}}$ in $M_{-}$such that $g$ is a critical metric on the (closed) domain $\Omega$ bounded by $\Sigma_{\zeta_{1}}$ and $\Sigma_{\zeta_{2}}$.

(iii) There exists a rotationally symmetric sphere $\Sigma_{\theta}$ in $M_{+}$(or equivalently $\left.M_{-}\right)$such that if $\Omega$ is the (closed) domain bounded by $\Sigma_{\theta}$ and $\Sigma_{0}$ and if $(\tilde{\Omega}, \tilde{g})$ is the quotient manifold obtained from $(\Omega, g)$ by identifying points on $\Sigma_{0}$ through the antipodal map on $\Sigma_{0}$, then $\tilde{g}$ is a critical metric on $\tilde{\Omega}$.

Corollary 3.2. Let $(M, g)$ be a complete, spatial Ads-Schwarzschild manifold with positive mass. Let $\Sigma_{0}$ be the horizon in $(M, g)$ (i.e. the unique closed minimal surface in $(M, g))$. The following are true:

(i) There exist functions $\lambda$ on $(M, g)$ satisfying (3.1).

(ii) Let $M_{+}, M_{-}$be the two components of $M \backslash \Sigma_{0}$. There exist a rotationally symmetric sphere $\Sigma_{\zeta}$ in $M_{+}$and a rotationally symmetric sphere $\Sigma_{-\zeta}$ in $M_{-}$, which is the image of $\Sigma_{\zeta}$ under the reflection with respect to $\Sigma_{0}$, such that if $U$ is the (closed) domain bounded by $\Sigma_{\zeta}$ and $\Sigma_{-\zeta}$, then for any rotationally symmetric sphere $\Sigma_{\zeta_{1}}$ in $M_{+} \backslash U$, there exists a rotationally symmetric sphere $\Sigma_{\zeta_{2}}$ in $M_{-} \backslash U$ such that $g$ is a critical metric on the (closed) domain $\Omega$ bounded by $\Sigma_{\zeta_{1}}$ and $\Sigma_{\zeta_{2}}$.

(iii) Let $U$ be as in (ii). There exists a rotationally symmetric sphere $\Sigma_{\theta}$ in $M_{+} \backslash U$ (or equivalently $\left.M_{-} \backslash U\right)$ such that if $\Omega$ is the (closed) domain bounded by $\Sigma_{\theta}$ and $\Sigma_{0}$ and if $(\tilde{\Omega}, \tilde{g})$ is the quotient manifold obtained from $(\Omega, g)$ by identifying points on $\Sigma_{0}$ through the antipodal map on $\Sigma_{0}$, then $\tilde{g}$ is a critical metric on $\tilde{\Omega}$.

We end this section with a discussion on the sign of the first Dirichlet eigenvalue of $(n-1) \Delta_{g}+R$ of those examples constructed in (1) and (2) with $R>0$.

Proposition 3.3. Let $R>0, a>0$ and $r$ be given as in Proposition 3.2(iii). Suppose $-s_{1}, 0$ and $s_{1}$ are three consecutive roots of $r^{\prime}$. Let $I$ be a finite closed interval in $\mathbb{R}^{1}$. Consider the manifold $\Omega=I \times N$ with the metric $g=d s^{2}+r^{2} h$, where $h$ is an Einstein metric on a closed manifold $N$ such that $\operatorname{Ric}(h)=(n-2) \kappa_{0} h$ with $\kappa_{0}$ satisfying (3.10).

(i) If $\left[0, s_{1}\right]$ is a proper subset of $I$, then the first Dirichlet eigenvalue of ( $n-$ 1) $\Delta_{g}+R$ on $(\Omega, g)$ is negative.

(ii) Let $\lambda$ be a solution to (3.11) on $\mathbb{R}^{1}$. Let $\zeta_{2} \in\left(-s_{1}, 0\right)$ and $\zeta_{1} \in\left(0, s_{1}\right)$ be the two consecutive roots of $\lambda$. Let $I=\left[\zeta_{2}, \zeta_{1}\right]$. Then the first Dirichlet eigenvalue of $(n-1) \Delta_{g}+R$ on $\Omega$ is positive if $r(0)=r_{\min }$ and is negative if $r(0)=r_{\max }$.

(iii) Suppose $r(0)=r_{\min }$. Let $\lambda_{0}$ be the even solution to (3.11) on $\mathbb{R}^{1}$. Let $-\theta \in\left(-s_{1}, 0\right)$ and $\theta \in\left(0, s_{1}\right)$ be the two consecutive roots of $\lambda_{0}$. Let $I=[-\theta, \theta]$. Let $(\Omega, g) / H$ be given as in (2). Then the first Dirichlet eigenvalue of $(n-1) \Delta_{g}+R$ on $(\Omega, g) / H$ is positive. 
Proof. (i) Note that (3.27) implies

$$
r^{\prime \prime \prime}+(n-1) \frac{r^{\prime} r^{\prime \prime}}{r}+\frac{R}{n-1} r^{\prime}=0,
$$

which implies

$$
\Delta_{g} r^{\prime}+\frac{R}{n-1} r=0
$$

on $\Omega$. Since $r^{\prime}(0)=r^{\prime}\left(s_{1}\right)=0$ and $r^{\prime}$ does not change sign in $\left(0, s_{1}\right)$, the first Dirichlet eigenvalue of $(n-1) \Delta_{g}+R$ on $\left(0, s_{1}\right) \times N$ must be zero. As $\left(0, s_{1}\right) \times N$ is a proper subset of $\Omega$, we conclude that (i) is true (see Lemma 1 in [6]).

(ii) By (3.7), we have

$$
\Delta_{g} \lambda+\frac{R}{n-1} \lambda=-\frac{n}{n-1}
$$

on $\Omega$. Let $\gamma$ be the first eigenvalue of $(n-1) \Delta_{g}+\frac{R}{n-1}$ on $\Omega$. Let $\phi$ be an eigenfunction satisfying

$$
\left\{\begin{aligned}
(n-1) \Delta_{g} \phi+\frac{R}{n-1} \phi+\gamma \phi & =0 \text { on } \Omega \\
\phi & =0 \text { on } \partial \Omega .
\end{aligned}\right.
$$

It follows from (3.47)-(3.48) and the fact $\lambda=0$ on $\partial \Omega$ that

$$
\gamma \int_{\Omega} \lambda \phi=\frac{n}{n-1} \int_{\Omega} \phi
$$

Since both $\phi$ and $\lambda$ do not change sign in the interior of $\Omega$, (3.49) implies that $\gamma$ has the same sign as $\lambda$ on $\left(-\zeta_{2}, \zeta_{1}\right)$. If $r(0)=r_{\min }$, we have $\lambda(0)>0$ by (iii) in Proposition 3.2, hence $\gamma>0$. Similarly, if $r(0)=r_{\max }$, we have $\lambda(0)<0$ and $\gamma<0$. Therefore, (ii) is proved.

(iii) follows directly from (ii) and the fact that the natural projection map from $(\Omega, g)$ to $(\Omega, g) / H$ is a local isometry.

\section{Conformally flat CRitical metrics}

In this section, we consider conformally flat metrics $g$ satisfying

$$
-\left(\Delta_{g} \lambda\right) g+\nabla_{g}^{2} \lambda-\lambda \operatorname{Ric}(g)=g
$$

for some function $\lambda$. Our main goal is to classify all compact manifolds with boundary which admit a conformally flat critical metric.

We start with local properties of such a metric. Similar to the work of Kobayashi and Obata in [9], we have the following:

Lemma 4.1. Let $\left(\Omega^{n}, g\right)$ be a connected, conformally flat Riemannian manifold. Suppose there exists a smooth function $\lambda$ such that $g$ and $\lambda$ satisfy (4.1). For $c \in \mathbb{R}$, let $N$ be a component of $\lambda>c$ which is the level set $\{\lambda=c\} \subset \Omega$ such that $\nabla \lambda \neq 0$ on $N$. Then the following hold:

(i) $|\nabla \lambda|$ is constant on $N$.

(ii) $N$ is totally umbilical with constant mean curvature.

(iii) $N$ has constant sectional curvature.

Proof. Let $R$ be the scalar curvature of $g$. By [11, $R$ equals a constant. The proof in [9] can then be carried over to our case. For the sake of completeness, we include 
the relevant details. First note that it is sufficient to consider the case that $c \neq 0$. Since $\Omega$ is conformally flat, we have (see [10] for example)

$$
\left(\nabla_{X} S\right)(Y, Z)-\left(\nabla_{Y} S\right)(X, Z)=0
$$

for all vector fields $X, Y, Z$, where $S$ is the Schouten tensor given by (at the points where $\lambda \neq 0$ )

$$
\begin{aligned}
(n-2) S & =\operatorname{Ric}(g)-\frac{R}{2(n-1)} g \\
& =\lambda^{-1} \nabla_{g}^{2} \lambda+\frac{1}{(n-1) \lambda} g+\frac{R}{2(n-1)} g
\end{aligned}
$$

where we have used (4.1). Moreover, the Weyl curvature tensor is zero and so the Riemannian curvature tensor of $g$ equals the Kulkarni-Nomizu product of $S$ and $g$, which together with (4.3) shows

$$
\begin{aligned}
R(X, Y, Z, U) & =\frac{R+2 \lambda^{-1}}{(n-1)(n-2)}[g(X, Z) g(Y, U)-g(X, U) g(Y, Z)] \\
& +\frac{1}{(n-2) \lambda}\left[\nabla^{2} \lambda(X, Z) g(Y, U)+\nabla^{2} \lambda(Y, U) g(X, Z)\right. \\
& \left.-\nabla^{2} \lambda(X, U) g(Y, Z)-\nabla^{2} \lambda(Y, Z) g(X, U)\right]
\end{aligned}
$$

for all vector fields $X, Y, Z, U$. Here $R(X, Y, Z, U)$ is defined as $\langle R(X, Y) U, Z\rangle$ with $R(X, Y)=\nabla_{X} \nabla_{Y}-\nabla_{Y} \nabla_{X}-\nabla_{[X, Y]}$.

By (4.2) and (4.3), we have

$$
\begin{aligned}
0= & D_{X}\left(\lambda^{-1} \nabla_{g}^{2} \lambda\right)(Y, Z)-D_{Y}\left(\lambda^{-1} \nabla_{g}^{2} \lambda\right)(X, Z)-\frac{X(\lambda) g(Y, Z)-Y(\lambda) g(X, Z)}{(n-1) \lambda^{2}} \\
= & \lambda^{-1}\left[D_{X}\left(\nabla_{g}^{2} \lambda\right)(Y, Z)-D_{Y}\left(\nabla_{g}^{2} \lambda\right)(X, Z)\right] \\
& -\lambda^{-2}\left(X(\lambda) \nabla^{2} \lambda(Y, Z)-Y(\lambda) \nabla^{2} \lambda(X, Z)\right) \\
& -\frac{1}{(n-1) \lambda^{2}}(X(\lambda) g(Y, Z)-Y(\lambda) g(X, Z)) \\
= & \lambda^{-1} R(X, Y, Z, \nabla \lambda)-\lambda^{-2}\left(X(\lambda) \nabla^{2} \lambda(Y, Z)-Y(\lambda) \nabla^{2} \lambda(X, Z)\right) \\
& -\frac{1}{(n-1) \lambda^{2}}(X(\lambda) g(Y, Z)-Y(\lambda) g(X, Z)) .
\end{aligned}
$$

Let $X$ be tangential to $N$ and $Z=Y=\nabla \lambda$; then we have

$$
0=Y(\lambda) \nabla^{2} \lambda(X, Z)=\frac{1}{2}|\nabla \lambda|^{2} X\left(|\nabla \lambda|^{2}\right)
$$

on $N$. Hence $|\nabla \lambda|$ is constant on $N$. This proves (i).

In (4.5), let $X, Z$ be tangential to $N, Y=\nabla \lambda$ and let $\xi=\nabla \lambda /|\nabla \lambda|$. Then we have

$$
R(X, \xi, Z, \xi)=-\lambda^{-1}\left(\nabla^{2} \lambda(X, Z)+\frac{1}{n-1} g(X, Z)\right)
$$


On the other hand, let $Y=U=\nabla \lambda$ and $X, Z$ be tangential to $N$ in (4.4). Then we have

$$
\begin{aligned}
R(X, \xi, Z, \xi)= & \frac{1}{(n-2)} \lambda^{-1}\left[\nabla^{2} \lambda(X, Z)+\nabla^{2} \lambda(\xi, \xi) g(X, Z)\right] \\
& +\frac{1}{(n-1)(n-2)}\left(2 \lambda^{-1}+R\right) g(X, Z) .
\end{aligned}
$$

Comparing (4.6) and (4.7), we have

$$
\begin{aligned}
(n-1) \nabla^{2} \lambda(X, Z) & =\left[-\nabla^{2} \lambda(\xi, \xi)-\frac{n+R \lambda}{n-1}\right] g(X, Z) \\
& =\left[-\nabla^{2} \lambda(\xi, \xi)+\Delta_{g} \lambda\right] g(X, Z),
\end{aligned}
$$

where the last step follows from

$$
\Delta_{g} \lambda=-\frac{1}{n-1}(R \lambda+n)
$$

which is obtained by taking the trace of (4.1). Recall

$$
\Delta_{g} \lambda=\Delta_{g}^{N} \lambda+H \frac{\partial \lambda}{\partial \xi}+\nabla^{2} \lambda(\xi, \xi)
$$

where $H$ is the mean curvature of $N$ and $\Delta_{g}^{N}$ is the Laplacian on $N$. Thus, (4.8) becomes

$$
|\nabla \lambda|^{-1} \nabla^{2} \lambda(X, Z)=\frac{H}{n-1} g(X, Z) .
$$

Now let $A(X, Z)=g\left(\nabla_{X} \xi, Z\right)$ be the second fundamental form of $N$. Then

$$
A(X, Z)=\frac{\nabla^{2} \lambda(X, Z)}{|\nabla \lambda|}=\frac{H}{n-1} g(X, Z),
$$

which shows $N$ is totally umbilical.

To prove that $H$ is constant on $N$, let $\alpha=H /(n-1)$. By (4.5) and the CodazziMainardi equation for $X, Y, Z$ tangential to $N$, we have

$$
\begin{aligned}
0 & =R(X, Y, Z, \xi) \\
& =\left(\nabla_{X}^{N} A\right)(Y, Z)-\left(\nabla_{Y}^{N} A\right)(X, Z) \\
& =X(\alpha) g(Y, Z)-Y(\alpha) g(X, Z),
\end{aligned}
$$

where $\nabla^{N}$ is the covariant derivative of $N$. For any given $X$, let $Y=Z$ be a unit vector perpendicular to $X$. Then $X(\alpha)=0$. Hence $\alpha$ is constant on $N$. This proves (ii).

To prove (iii), let $X=Z$ and $Y=U$ in (4.4) and choose $X$ and $Y$ to be orthonormal tangent vectors tangent to $N$. It follows from (4.4), (4.10) and the fact $|\nabla \lambda|$ and $H$ are constant on $N$ that $R(X, Y, X, Y)$ is constant on $N$. By the Gauss equation and (ii), we conclude that $N$ has constant sectional curvature.

In the rest of this section, we assume that $(\Omega, g)$ is a connected, compact Riemannian manifold with a smooth (possibly disconnected) boundary $\Sigma$. Moreover, we make the following assumption on $(\Omega, g)$ :

Assumption A. $\left(\Omega^{n}, g\right)$ is conformally flat and there is a smooth function $\lambda$ satisfying (4.1) and vanishing on $\Sigma$. Furthermore, the first Dirichlet eigenvalue of $(n-1) \Delta_{g}+R$ is non-negative. 
Note that the condition on the first Dirichlet eigenvalues is automatically satisfied if $R \leq 0$.

Given such an $(\Omega, g)$, by [1] we have $\lambda>0$ in the interior of $\Omega$. In addition, if $\nu$ denotes the outward unit normal to $\Sigma$, then $\frac{\partial \lambda}{\partial \nu}<0$ and is constant on each connected component of $\Sigma$. Similar to [9], we can now prove the following result.

Lemma 4.2. Let $\Sigma_{0}$ be a connected component of $\Sigma$. Let $\widetilde{\Omega}_{0}$ be the connected component of the open set $\{|\nabla \lambda|>0\}$ in $\Omega$ such that its closure contains $\Sigma_{0}$, and let $\Omega_{0}=\widetilde{\Omega}_{0} \cup \Sigma_{0}$. Then there exists a constant $\delta_{0}>0$ such that $\left(\Omega_{0}, g\right)$ is isometric to a warped product $\left(\left[0, \delta_{0}\right) \times \Sigma_{0}, d s^{2}+r^{2} h\right)$, where $r>0$ is a smooth function on $\left[0, \delta_{0}\right)$ and $h$ is the induced metric on $\Sigma_{0}$ from $g$. Moreover, $\lambda$ on $\Omega_{0}$ depends only on $s \in\left[0, \delta_{0}\right)$, and $\Sigma_{0}$ has constant sectional curvature.

Proof. The claim that $\Sigma_{0}$ has constant sectional curvature is a direct corollary of Lemma 4.1 and the facts $\lambda=0$ on $\Sigma_{0}$ and $\frac{\partial \lambda}{\partial \nu} \neq 0$ on $\Sigma_{0}$. On $\Omega_{0}$, define the smooth vector field $v=\nabla \lambda /|\nabla \lambda|^{2}$. This vector field is smooth up to $\Sigma_{0}$. For any $x \in \Sigma_{0}$, let $\zeta_{x}(s)$ be the integral curve of $v$ such that $\zeta_{x}(0)=x$. Then $\zeta_{x}$ can be extended until it meets the boundary of $\Omega_{0}$. Suppose $\zeta_{x}$ is defined on $\left[0, \delta_{x}\right)$; then

$$
\lambda\left(\zeta_{x}(s)\right)=\int_{0}^{s} g\left(\nabla \lambda\left(\zeta_{z}(\tau)\right), \zeta_{x}^{\prime}(\tau) g d \tau+\lambda(x)=s .\right.
$$

Hence if $\left[0, \delta_{x}\right)$ is the maximal domain of the definition of $\zeta_{x}$ and $\max _{\Omega} \lambda$ is the maximum value of $\lambda$ on $\Omega$, then $\delta_{x} \leq \max _{\Omega} \lambda<\infty$. Note that $\lambda\left(\zeta_{x}(s)\right)$ is increasing in $s$ and $\frac{\partial \lambda}{\partial \nu}<0$ on $\Sigma$. It is easily seen that for any $s_{i} \rightarrow \delta_{x}, \zeta_{x}\left(s_{i}\right)$ cannot converge to a point at $\Sigma$.

We claim that $\delta_{x}$ is constant on $\Sigma_{0}$. It is sufficient to prove that $\delta_{x}=\delta$ where $\delta=\inf _{y \in \Sigma_{0}} \delta_{y}$, which is positive as $\Sigma_{0}$ is compact. Suppose $\delta_{x}>\delta$ for some $x \in \Sigma_{0}$. Then $|\nabla \lambda| \geq c>0$ on $\zeta_{x}(\delta-\epsilon, \delta+\epsilon)$ for some constants $c$ and $\epsilon>0$. For any $s \in(0, \delta)$, let $N_{s}=\left\{\zeta_{y}(s) \mid y \in \Sigma_{0}\right\}$. Then $|\nabla \lambda|>0$ on $N_{s}$, and $\lambda=s$ on $N_{s}$ by (4.13). Moreover, $N_{s}$ is connected as $\Sigma_{0}$ is connected. Therefore, Lemma 4.1 implies that $|\nabla \lambda|$ is constant on $N_{s}$. Consequently, $|\nabla \lambda| \geq c$ on $N_{s}$ for all $s \in(\delta-\epsilon, \delta)$. This implies that all $\zeta_{y}$ can be extended up to $\delta+\epsilon^{\prime}$ for some $\epsilon^{\prime}>0$ independent of $y$, which contradicts the definition of $\delta$. Hence $\delta_{x}=\delta$ for all $x \in \Sigma_{0}$.

Let $I=[0, \delta)$ and define the map $\Phi: I \times \Sigma_{0} \rightarrow \Omega_{0}$ by $\Phi(s, x)=\zeta_{x}(s)$. Then $\Phi$ is an injective, local diffeomorphism. It is also true that $\Phi\left(I \times \Sigma_{0}\right)$ is closed in $\Omega_{0}$ because if $x_{k} \in \Phi\left(I \times \Sigma_{0}\right)$ with $x_{k} \rightarrow x \in \Omega_{0}$ and if $x \notin \Phi\left(I \times \Sigma_{0}\right)$, then $\nabla \lambda(x)=0$, contradicting the definition of $\Omega_{0}$. Since $\Omega_{0}$ is connected, we conclude $\Omega_{0}=\Phi\left(I \times \Sigma_{0}\right)$.

Let $\left(u_{1}, \ldots, u_{n-1}\right)$ be some local coordinates on $\Sigma_{0}$. Then $\Phi_{*}\left(\partial_{s}\right)=\vec{v}$ is orthogonal to $\Phi_{*}\left(\partial_{u_{i}}\right)$. Writing $\Phi_{*}\left(\partial_{u_{i}}\right)$ as $\partial_{u_{i}}$, we have

$$
\begin{aligned}
\frac{\partial}{\partial s} g\left(\partial_{u_{i}}, \partial_{u_{j}}\right) & =g\left(\nabla_{\partial_{u_{i}}} \vec{v}, \partial_{u_{j}}\right)+g\left(\partial_{u_{i}}, \nabla_{\partial_{u_{j}}} \vec{v}\right) \\
& =2|\nabla \lambda|^{-1} \operatorname{II}\left(\partial_{u_{i}}, \partial_{u_{j}}\right)
\end{aligned}
$$

where II is the second fundamental form of $N_{s}$ with respect to $\vec{v}$. By Lemma 4.1. $\mathrm{II}\left(\partial_{u_{i}}, \partial_{u_{j}}\right)=\alpha g\left(\partial_{u_{i}}, \partial_{u_{j}}\right)$ for some function $\alpha$ depending only on $s$; moreover, $|\nabla \lambda|$ also depends only on $s$. Therefore, in terms of coordinates $\left(s, u_{1}, \ldots, u_{n-1}\right)$ on $\Omega_{0}$, the metric $g$ can be written as

$$
g=|\nabla \lambda|^{-2} d s^{2}+\beta h
$$


where $\beta$ is a function of $s$ and $h$ is the induced metric on $\Sigma_{0}$. Rescaling $s$ using the fact that $|\nabla \lambda|$ depends only on $s$, we may re-write $g$ as $g=d s^{2}+r^{2} h$, where $s \in\left[0, \delta_{0}\right)$ for some $\delta_{0}$ possibly different from $\delta$, and $r$ is some function depending only on $s$. The fact $\delta_{0}<+\infty$ follows from the assumption that $\Omega$ is compact.

Let $\Sigma_{0}, I=\left[0, \delta_{0}\right), \Omega_{0}, r$ and $h$ be given as in Lemma 4.2, We identify $I \times \Sigma_{0}$ with $\Omega_{0}$ using the isometry. Since $\frac{\partial \lambda}{\partial \nu}<0$ on $\Sigma_{0}$ and $|\nabla \lambda|>0$ on $I \times \Sigma_{0}$, we have $\lambda^{\prime}(s)>0$ on $I \times \Sigma_{0}$, where " $"$ " denotes the derivative w.r.t. $s$. For convenience, we also normalize $R$ so that $R=n(n-1) \kappa$ with $\kappa=0,1$ or -1 . By Proposition 3.1 in Section 3, we have

$$
r^{\prime \prime}+\kappa r=a r^{1-n}
$$

for some constant $a$, and

$$
\frac{r^{\prime}}{r} \lambda^{\prime}-\frac{r^{\prime \prime}}{r} \lambda=-\frac{1}{n-1} .
$$

Also from Section 2, we have

$$
\operatorname{Ric}(g)\left(\partial_{s}, \partial_{s}\right)=-(n-1) \frac{r^{\prime \prime}}{r}=(n-1) \kappa-(n-1) a r^{-n}
$$

and

$$
\left(r^{\prime}\right)^{2}+\kappa r^{2}+\frac{2 a}{n-2} r^{2-n}=\kappa_{0},
$$

where $\kappa_{0}$ is the sectional curvature of $\left(\Sigma_{0}, h\right)$ which is a constant.

In what follows, we let $\overline{I \times \Sigma_{0}}$ be the closure of $I \times \Sigma_{0}$ in $\Omega \cup \Sigma_{0}$. Since $\nabla \lambda=0$ somewhere in $\Omega, \overline{I \times \Sigma_{0}} \backslash I \times \Sigma_{0}$ is not empty and consists of points at which $\nabla \lambda=0$.

Lemma 4.3. With the above notation, the following are true:

(i) $a \geq 0$.

(ii) If $a=0$, then $(\Omega, g)$ is a geodesic ball in space forms.

(iii) If $a>0$, then $S_{0}=\overline{I \times \Sigma_{0}} \backslash I \times \Sigma_{0}$ is a connected, embedded, totally umbilical hypersurface with constant mean curvature in $\Omega$. Moreover, for any $p \in S_{0}$ there is an open neighborhood $U$ of $p$ such that $U \cap S_{0}=U \cap S$, where $S=\{q \in \Omega \mid \nabla \lambda(q)=0\}$.

Proof. (i) Suppose $a<0$. By (4.16), we have $\liminf _{s} \nearrow \delta_{0} r(s)>0$. Suppose there exists $s_{k} \nearrow \delta_{0}$ such that $r\left(s_{k}\right) \rightarrow \infty$; then (4.17) implies

$$
\kappa+\left(\frac{r^{\prime}\left(s_{k}\right)}{r\left(s_{k}\right)}\right)^{2} \rightarrow 0 .
$$

In particular, $\left(\frac{r^{\prime}\left(s_{k}\right)}{r\left(s_{k}\right)}\right)^{2}$ are uniformly bounded. Since $\lambda^{\prime}\left(s_{k}\right) \rightarrow 0$, by (4.14) and (4.15) we have

$$
-\kappa \lim _{k \rightarrow \infty} \lambda\left(s_{k}\right)=\frac{1}{n-1},
$$

which is impossible if $\kappa=0,1$ because $\lambda>0$ in the interior of $\Omega$. Suppose $\kappa=-1$. By (4.14), $r^{\prime \prime}<r$. Let $f$ be a function on $I$ such that $f^{\prime \prime}=f, f(0)=r(0)$ and $f^{\prime}(0)>r^{\prime}(0)$. Then $f>r$ near 0 . Since $f$ is bounded on $I$ and $r\left(s_{k}\right) \rightarrow \infty$, there exists $s_{0}>0$ such that $f>r$ on $\left(0, s_{0}\right)$ and $f\left(s_{0}\right)=r\left(s_{0}\right)$. So we have $(r-f)^{\prime \prime}<(r-f), r-f<0$ on $\left(0, s_{0}\right)$, but $r-f=0$ at $0, s_{0}$. This is impossible. 
Hence, we have $\lim \sup _{s \nearrow \delta_{0}} r(s)<+\infty$. It follows that $C^{-1} \leq r \leq C$ on $I$ for some $C>0$. In particular, $r$ can be extended smoothly beyond $\delta_{0}$ satisfying (4.14), and $\lambda$ can be extended smoothly beyond $\delta_{0}$ satisfying (4.15). At $\delta_{0}$, we have $\lambda^{\prime}\left(\delta_{0}\right)=0$, hence (4.14) and (4.15) imply

$$
\lambda\left(\delta_{0}\right)=\frac{1}{n-1} \cdot \frac{1}{-\kappa+a r^{-n}\left(\delta_{0}\right)} .
$$

Again this is impossible if $\kappa=0,1$. Suppose $\kappa=-1$; then $\lambda\left(\delta_{0}\right)>\frac{1}{n-1}$. Recall that $\lambda=0$ on $\Sigma$ and by (4.9) we have

$$
\Delta_{g}\left(\lambda-\frac{1}{n-1}\right)-n\left(\lambda-\frac{1}{n-1}\right)=0 \text { on } \Omega .
$$

Hence, $\max _{\Omega} \lambda \leq \frac{1}{n-1}$, contradicting $\lambda\left(\delta_{0}\right)>\frac{1}{n-1}$. This proves (i).

(ii) Suppose $a=0$. Then (4.14) becomes $r^{\prime \prime}+\kappa r=0$. Hence $r$ can be defined for all $s$. In particular, $\lim _{s \rightarrow \delta} r(s)=r_{0}$ exists. Suppose $r_{0}>0$; then $\lambda$ can be extended beyond $\delta$ satisfying (4.15). As in case (i), it follows from (4.14) and (4.15) that

$$
-\kappa \lambda\left(\delta_{0}\right)=\frac{1}{n-1}
$$

which is impossible if $\kappa=0$ or 1 . If $\kappa=-1$, then $\lambda\left(\delta_{0}\right)=\frac{1}{n-1}$. However, by the proof of (i), we have $\max _{\Omega} \lambda \leq \frac{1}{n-1}$. Thus, the function $\lambda-\frac{1}{n-1}$, which is not a constant, achieves an interior maximum which is zero. By (4.19), we get a contradiction to the strong maximum principle. Therefore, $r_{0}=0$. Consequently, $\overline{I \times \Sigma_{0}} \backslash I \times \Sigma_{0}$ consists of only one point, say $p$. Let $B_{p} \subset \Omega$ be a connected, open neighborhood of $p$. Then $\left(B_{p} \backslash\{p\}\right) \cap\left(I \times \Sigma_{0}\right)$ and $\left(B_{p} \backslash\{p\}\right) \backslash \overline{I \times \Sigma_{0}}$ are both open sets in $B_{p} \backslash\{p\}$. Hence $\left(B_{p} \backslash\{p\}\right) \backslash \overline{I \times \Sigma_{0}}=\emptyset$. As $\Omega$ is connected, we conclude that $\Omega=\overline{I \times \Sigma_{0}}$. As $a=0$, by Remark 3.1 the metric $g$ is Einstein. Hence $(\Omega, g)$ is a geodesic ball in space forms by Theorem 2.1.

(iii) Suppose $a>0$. By Lemma 3.3, $r$ can be extended to be a solution on $\mathbb{R}$ and is bounded below away from zero. Hence $r$ satisfies $C^{-1} \leq r \leq C$ in $\left[0, \delta_{0}\right)$ for some positive constant $C$.

For each $y \in \Sigma_{0}$, let $\alpha_{y}(s)$ denote the geodesic starting from $y$ with $\alpha_{y}^{\prime}(0)=\partial_{s}$. As $\Sigma_{0}$ is compact, there exists $\delta_{1}>\delta_{0}$ such that $\alpha_{y}(s)$ is defined on $\left[0, \delta_{1}\right)$ for all $y \in \Sigma_{0}$. Clearly, the set $\left\{\alpha_{y}\left(\delta_{0}\right) \mid y \in \Sigma_{0}\right\}$ is contained in $S_{0}$. On the other hand, for any $p \in S_{0}$ there exists $\left(s_{k}, x_{k}\right) \in I \times \Sigma_{0}$ such that $\alpha_{x_{k}}\left(s_{k}\right) \rightarrow p$ with $s_{k} \rightarrow \delta_{0}$. As $\Sigma_{0}$ is compact and $r \leq C$, there exists $x \in \Sigma_{0}$ such that $\alpha_{x}\left(s_{k}\right) \rightarrow p$. Hence, $p=\alpha_{x}\left(\delta_{0}\right)$. This shows $S_{0}=\left\{\alpha_{y}\left(\delta_{0}\right) \mid y \in \Sigma_{0}\right\}$; in particular, $S_{0}$ is connected (as $\Sigma_{0}$ is connected).

To show $S_{0}$ is an embedded hypersurface, we let $\Sigma_{s}=\{s\} \times \Sigma_{0}$ for each $0<$ $s<\delta_{0}$. Since the induced metric on $\Sigma_{s}$ is $r^{2} h$ and $r \geq C^{-1}$, the curvature of $\Sigma_{s}$ is bounded by a constant independent of $s$. Since $\Sigma_{s}$ is totally umbilical, it follows from the Gauss equation that the norm of the second fundamental form of $\Sigma_{s}$ is also bounded by a constant independent of $s$. For any $p=\alpha_{x}\left(\delta_{0}\right) \in S_{0}$, using the estimates of Lemma A.2 in the Appendix and the fact that $\Sigma_{s}$ is of constant mean curvature with uniformly bounded second fundamental form, we conclude that there exists $\rho>0$ and a sequence $s_{k} \nearrow \delta_{0}$ such that $N_{k}=\left\{\left(s_{k}, y\right) \mid y \in\right.$ $\left.B_{0}(x, \rho)\right\}$ converges to an embedded hypersurface $N_{p}$ passing through $p$. Here $B_{0}(x, \rho)$ denotes a geodesic ball in $\left(\Sigma_{0}, h\right)$ centered at $x$ with radius $\rho$. Note that $N_{p} \subset S_{0}$. 
At $p=\alpha_{x}\left(\delta_{0}\right)$, we have $\nabla \lambda=0$. By (4.1) and (4.16), we have

$$
\nabla_{g}^{2} \lambda\left(\alpha_{x}^{\prime}\left(\delta_{0}\right), \alpha_{x}^{\prime}\left(\delta_{0}\right)\right)=-\kappa \lambda-\frac{1}{n-1}-(n-1) a r^{-n} .
$$

As $a>0$, (4.20) implies $\nabla_{g}^{2} \lambda\left(\alpha_{x}^{\prime}\left(\delta_{0}\right), \alpha_{x}^{\prime}\left(\delta_{0}\right)\right)<0$. This is obvious if $\kappa=0$ or 1 . If $\kappa=-1$, this follows from the fact $\max _{\Omega} \lambda<\frac{1}{n-1}$.

By shrinking $B_{0}(x, \rho)$ if necessary, we may assume that there exist small positive constants $b$ and $c$ such that $\nabla_{g}^{2} \lambda\left(\alpha_{y}^{\prime}(s), \alpha_{y}^{\prime}(s)\right)<-c$ for all $y \in B_{0}(x, \rho)$ and $s \in$ $\left(\delta_{0}-b, \delta_{0}+b\right)$. As $\nabla \lambda=0$ at $\alpha_{y}\left(\delta_{0}\right) \in S_{0}$, we have $g\left(\nabla \lambda, \alpha_{y}^{\prime}(s)\right) \neq 0$ for all $y \in B_{0}(x, \rho)$ and $s \in\left(\delta_{0}-b, \delta_{0}+b\right)$. In particular, $\nabla \lambda$ is not zero at the points $\alpha_{y}(s)$ for all such $y$ and $s$.

We want to show that there is an open neighborhood $U$ of $p$ such that $U \cap S=$ $U \cap N_{p}$. If not, then there exists a sequence of points $\left\{p_{k}\right\} \subset \Omega$ such that $p_{k} \rightarrow p$, $p_{k} \notin N_{p}$ and $p_{k} \in S$. For $k$ sufficiently large, there exist minimal geodesics $\beta_{p_{k}}(t)$ starting from $p_{k}$ and ending at $N_{p}$ so that $\beta_{p_{k}}^{\prime}(t)$ is perpendicular to $N_{p}$ at some point $q_{k}=\alpha_{y_{k}}\left(\delta_{0}\right)$ for some $y_{k} \in B_{0}(x, \rho)$. Since $\beta_{p_{k}}$ and $\alpha_{y_{k}}$ are two geodesics both perpendicular to $N_{p}$ at $q_{k}$, we must have $p_{k}=\alpha_{y_{k}}\left(s_{k}\right)$ for some $s_{k}$. Moreover, $s_{k} \neq \delta_{0}$ as $p_{k} \notin N_{k}$. When $k$ is sufficiently large, we have $s_{k} \in\left(\delta_{0}-b, \delta_{0}+b\right)$. Hence $\nabla \lambda$ is not zero at $p_{k}=\alpha_{y_{k}}\left(s_{k}\right)$, contradicting the fact that $p_{k} \in S$.

As $S_{0} \subset S$, we conclude that $S_{0}$ is an embedded hypersurface in $\Omega$ such that for each $p \in S_{0}$ there is an open neighborhood $U$ of $p$ such that $U \cap N_{p}=U \cap S$. The fact that $S_{0}$ is totally umbilical and has constant mean curvature follows directly from the fact that each $\Sigma_{s}$ is totally umbilical and has constant mean curvature.

Let $(\Omega, g)$ be given as before. Assume that $(\Omega, g)$ is not a geodesic ball in space forms. Let $\Sigma_{1}, \ldots, \Sigma_{k}$ be the connected components of the boundary $\Sigma$. For each $i=1, \ldots, k$, let $\Omega_{i}$ be the connected component of the open set $\{|\nabla \lambda|>0\}$ in $\Omega$ whose closure contains $\Sigma_{i}$. By Lemma 4.2. each $\Omega_{i}$ can then be identified with $I_{i} \times \Sigma_{i}$ where $I_{i}=\left(0, \delta_{i}\right)$ for some $0<\delta_{i}<\infty$. On $I_{i} \times \Sigma_{i}$, the metric $g$ has the form $d s^{2}+r_{i}^{2} h_{i}$, where $r_{i}$ is some smooth positive function on $I_{i}$. By (4.14) and Lemma 4.3, each $r_{i}$ satisfies $r_{i}^{\prime \prime}+\kappa r=a_{i} r^{1-n}$ for some constant $a_{i}>0$. Let $\overline{I_{i} \times \Sigma_{i}}$ be the closure of $I_{i} \times \Sigma_{i}$ in $\Omega$ and let $S_{i}=\overline{I_{i} \times \Sigma_{i}} \backslash I_{i} \times \Sigma_{i}$. By Lemma 4.3, each $S_{i}$ is a connected, embedded, totally umbilical hypersurface with constant mean curvature in the interior of $\Omega$.

Lemma 4.4. With the above assumptions and notation, $\Sigma$ has at most two connected components, i.e. $k \leq 2$. If $k=2$, then $S_{1}=S_{2}$ and $\Omega=\overline{I_{1} \times \Sigma_{1}} \cup \overline{l_{2} \times \Sigma_{2}}$. If $k=1$, then $\Omega=\overline{I_{1} \times \Sigma_{1}}$.

Proof. Suppose $k \geq 2$ and suppose $S_{i} \cap S_{j} \neq \emptyset$ for some $i \neq j$, say $i=1, j=2$. For any $p \in S_{1} \cap S_{2}$, Lemma 4.3 implies there exists an open neighborhood $U$ of $p$ in $\Omega$ such that $U \cap S_{1}=U \cap S$, where $S=\{\nabla \lambda=0\}$. As $S_{2} \subset S$, we have $U \cap S_{2} \subset U \cap S_{1}$. As $S_{1}$ and $S_{2}$ are embedded hypersurfaces, the above implies $S_{1} \cap S_{2}$ is an open subset of both $S_{1}$ and $S_{2}$. As $S_{1}$ and $S_{2}$ are connected, we have $S_{1}=S_{1} \cap S_{2}=S_{2}$. Now, every geodesic in $\Omega$ emanating from and perpendicular to $S_{1}=S_{2}$ is either contained in $I_{1} \times \Sigma_{1}$ or $I_{2} \times \Sigma_{2}$. Hence, $\overline{I_{1} \times \Sigma_{1}} \cup \overline{I_{2} \times \Sigma_{2}}$ is both an open and a closed set in $\Omega$. As $\Omega$ is connected, we must have $\Omega=\overline{I_{1} \times \Sigma_{1}} \cup \overline{I_{2} \times \Sigma_{2}}$ and $k=2$.

Suppose $k \geq 2$ and suppose $S_{i} \cap S_{j}=\emptyset$ for any $i \neq j$. We prove that this is impossible by considering $U=\Omega \backslash \bigcup_{i} \overline{I_{i} \times \Sigma_{i}}$. If $U=\emptyset$, then each $\overline{I_{i} \times \Sigma_{i}}$ would 
be both open and closed in $\Omega$, contradicting the fact that $\Omega$ is connected. Suppose $U \neq \emptyset$. If $\kappa=0$ or 1 , then (4.9) implies

$$
\Delta_{g} \lambda=-n \kappa \lambda-\frac{n}{n-1}<0,
$$

where we also used $\lambda>0$ in the interior of $\Omega$. Hence, $\min _{\bar{U}} \lambda$ could only occur at $\partial U=\bigcup_{i} S_{i}$. Suppose $p \in \partial U$ such that $\lambda(p)=\min _{\bar{U}} \lambda$. Then the strong maximum principle implies $\frac{\partial \lambda}{\partial \nu_{U}} \neq 0$, where $\nu_{U}$ is a unit normal vector to $\partial U$ at $p$. This contradicts the fact that $\nabla \lambda=0$ at points in $S_{i}$. If $\kappa=-1$, then as in the proof of Lemma 4.3 we have $\lambda<\frac{1}{n-1}$ on $\Omega$ and

$$
\Delta_{g}\left(\lambda-\frac{1}{n-1}\right)=n\left(\lambda-\frac{1}{n-1}\right) \text {. }
$$

Applying the strong maximum principle to $\lambda-\frac{1}{n-1}$ on $U$, we get a contradiction as before. Therefore, we conclude that if $k \geq 2$, then $k=2, S_{1}=S_{2}$ and $\Omega=$ $\overline{I_{1} \times \Sigma_{1}} \cup \overline{l_{2} \times \Sigma_{2}}$.

Next, suppose $k=1$. Let $U=\Omega \backslash \overline{I_{1} \times \Sigma_{1}}$. The exact same argument in the previous paragraph implies $U=\emptyset$. We conclude $\Omega=\overline{I_{1} \times \Sigma_{1}}$.

Theorem 4.1. Let $(\Omega, g)$ be a connected, compact Riemannian manifold with a disconnected boundary $\Sigma$. Suppose $(\Omega, g)$ satisfies Assumption A. Then $(\Omega, g)$ is one of the manifolds constructed in Example (1) after Proposition 3.2.

Proof. By Lemma 4.4 $\Sigma$ has exactly two connected components, say $\Sigma_{1}$ and $\Sigma_{2}$. Moreover, if $I_{i}=\left[0, \delta_{i}\right), I_{i} \times \Sigma_{i}, \overline{I_{i} \times \Sigma_{i}}$ and $S_{i}$ are given as in Lemma 4.4 for $i=1$, 2 , then $S_{1}=S_{2}$ and $\Omega=\overline{I_{1} \times \Omega_{1}} \cup \overline{I_{2} \times \Omega_{2}}$. On each $I_{i} \times \Sigma_{i}$, by Lemmas 4.2 and 4.3 the metric $g$ has the form

$$
g=d s^{2}+r_{i}^{2} h_{i},
$$

where $h_{i}$ is a metric on $\Sigma_{i}$ with constant sectional curvature and $r_{i}$ is a smooth positive function on $I_{i}$ satisfying

$$
r_{i}^{\prime \prime}+\kappa r_{i}=a_{i} r_{i}^{1-n}
$$

for some constant $a_{i}>0$. Here we normalized $g$ so that its scalar curvature, which is a constant, is $n(n-1) \kappa$ with $\kappa=0$ or \pm 1 . Note that (4.21) and (4.22) are invariant if the triple $\left(r_{i}, h_{i}, a_{i}\right)$ is replaced by $\left(c r_{i}, c^{-2} h_{i}, c^{n} a\right)$ for any $c>0$. Hence, after rescaling, we may assume that $a_{1}=a_{2}$.

Let $S$ denote $S_{1}=S_{2}$. For any $p \in S$, there exists $x \in \Sigma_{1}, y \in \Sigma_{2}$ such that $\alpha_{x}\left(\delta_{1}\right)=p=\beta_{y}\left(\delta_{2}\right)$. Here $\alpha_{x}(s), \beta_{y}(s)$ denote the geodesic staring from $x$, $y$ with $\alpha_{x}^{\prime}(0)=\partial_{s}, \beta_{y}^{\prime}(0)=\partial_{s}$, respectively. At $p, \alpha_{x}^{\prime}\left(\delta_{1}\right)$ and $\beta_{y}^{\prime}\left(\delta_{2}\right)$ are both perpendicular to $S$. Hence $\alpha_{x}^{\prime}\left(\delta_{1}\right)=-\beta_{y}^{\prime}\left(\delta_{2}\right)$, and $\gamma=\alpha_{x} \cup\left(-\beta_{y}\right)$ is a geodesic in $\Omega$, where $-\beta_{y}(s)$ is defined as $\beta_{y}(-s)$. At $p$, recall that $\nabla \lambda=0$. By (4.15), we then have

$$
\frac{r_{1}^{\prime \prime}\left(\delta_{1}\right)}{r_{1}\left(\delta_{1}\right)}=\frac{r_{2}^{\prime \prime}\left(\delta_{2}\right)}{r_{2}\left(\delta_{2}\right)}
$$

It follows from (4.22), (4.23) and the fact $a_{1}=a_{2}$ that $r_{1}\left(\delta_{1}\right)=r_{2}\left(\delta_{2}\right)$. On the other hand, using the fact that the mean curvature of $S$ w.r.t. $\alpha_{x}^{\prime}\left(\delta_{1}\right)$ is negative of the mean curvature of $S$ w.r.t. $\beta_{y}^{\prime}\left(\delta_{2}\right)$, we conclude $r_{1}^{\prime}\left(\delta_{1}\right)=-r_{2}^{\prime}\left(\delta_{2}\right)$. In particular, if 
we let $I=\left[0, \delta_{1}+\delta_{2}\right]$ and define $r(s)=r_{1}(s), s \in\left[0, \delta_{1}\right]$ and $r(s)=r_{2}\left(\delta_{1}+\delta_{2}-s\right), s \in$ $\left[\delta_{1}, \delta_{1}+\delta_{2}\right]$, then $r(s)$ is a smooth function on $I$ satisfying

$$
r^{\prime \prime}+\kappa r=a r^{1-n},
$$

where $a=a_{1}=a_{2}$ is some positive constant.

Now suppose there exists another $\tilde{x} \in \Sigma_{1}$ such that $\alpha_{\tilde{x}}\left(\delta_{1}\right)=p$. Then we would have $\alpha_{\tilde{x}}^{\prime}\left(\delta_{1}\right)=\alpha_{x}^{\prime}\left(\delta_{1}\right)$, hence $\tilde{x}=x$. This implies the maps $x \mapsto \alpha_{x}\left(\delta_{1}\right), y \mapsto \beta_{y}\left(\delta_{2}\right)$ are bijective from $\Sigma_{1}, \Sigma_{2}$ to $S$. Consequently, the map $(x, s) \mapsto \gamma_{x}(s)$, where $\gamma_{x}(s)$ is the geodesic starting from $x \in \Sigma_{1}$ and perpendicular to $\Sigma$, is a diffeomorphism from $I \times \Sigma_{1}$ to $\Omega$. By (4.21), the induced metric from $g$ on $S=\left\{\delta_{1}\right\} \times \Sigma_{1}$ is given by both $r_{1}^{2}\left(\delta_{1}\right) h_{1}$ and $r_{2}^{2}\left(\delta_{2}\right) h_{2}$. As $r_{1}\left(\delta_{1}\right)=r_{2}\left(\delta_{2}\right)$, we have $h_{1}=h_{2}$.

Note that $r^{\prime}(0)=r_{1}^{\prime}(0)<0$ and $r^{\prime}\left(\delta_{1}+\delta_{2}\right)=-r_{2}^{\prime}(0)>0$; hence there exists an $s_{0} \in I$ such that $r^{\prime}\left(s_{0}\right)=0$. Replacing $s$ by $s-s_{0}$, we conclude that $\Omega$ is isometric to $I \times \Sigma_{1}$, where $I$ is replaced by $\left(-s_{0}, \delta_{1}+\delta_{2}-s_{0}\right)$ and the metric $g$ is given by $g=d s^{2}+r^{2} h$ with $r$ satisfying (4.24) and $r^{\prime}(0)=0$. Moreover, $\lambda$ only depends on $s \in I$. As $a>0$, by Section 2 we know that both $r$ and $\lambda$ can be extended to $\mathbb{R}^{1}$ and 0. Therefore, $(\Omega, g)$ is one of the examples in Example (1) after Proposition 3.2 .

Theorem 4.2. Let $(\Omega, g)$ be a connected, compact Riemannian manifold with a connected boundary $\Sigma$. Suppose $(\Omega, g)$ satisfies Assumption A. Then $(\Omega, g)$ is either a geodesic ball in a simply connected space form or one of the manifolds constructed in Example (2) after Proposition 3.2.

Proof. Suppose that $(\Omega, g)$ is not a geodesic ball in a simply connected space form. Since the boundary $\Sigma$ is connected, by Lemmas $4.2,4.3$ and 4.4 we have $\Omega=\overline{I \times \Sigma}$ (closure is taken with respect to $\Omega$ ) with the metric $g$ on $I \times \Sigma$ given by $d s^{2}+r^{2}(s) h$, where $I=[0, \delta)$ for some positive number $\delta$. Now the functions $r$ and $\lambda$ satisfy (4.14) (with $a>0$ ) and (4.15). Moreover, $S=\overline{I \times \Sigma} \backslash I \times \Sigma$ is a connected, embedded hypersurface in the interior of $\Omega$. Let $\left(U ; x^{1}, \ldots, x^{n}\right)$ be a local coordinate in $\Omega$ such that $U \cap S=\left\{x^{n}=0\right\}$. Let $U_{+}=\left\{x \in U \mid x^{n}>0\right\}$ and $U_{-}=\left\{x \in U \mid x^{n}<0\right\}$. Since $\Omega \backslash S=I \times \Sigma$, both $U_{+}$and $U_{-}$are contained in $I \times \Sigma$. In particular, as $s \nearrow \delta$, the surfaces $(\{s\} \times \Sigma) \cap U_{+}$and $(\{s\} \times \Sigma) \cap U_{-}$converge to $S \cap U$ from two sides of $S \cap U$ in $U$. As the mean curvature $H_{s}$ of $\{s\} \times \Sigma$ is constant for each $s \in I$, the mean curvature $H$ of $S \cap U$ is given by both $\lim _{s \rightarrow \delta_{-}} H_{s}$ and $-\lim _{s \rightarrow \delta_{-}} H_{s}$. Hence, $H=0$. Since $S$ is totally umbilical with constant mean curvature by Lemma 4.3, we conclude that $S$ is totally geodesic.

Now consider $\tilde{M}=[0, \delta] \times \Sigma$ with the metric $\tilde{g}=d s^{2}+r^{2} h$ (the fact $a>0$ implies that $r$ is smooth up to $\delta$ with $r(\delta)>0)$. Let $D \tilde{M}$ denote the doubling of $(\tilde{M}, \tilde{g})$ with respect to $\Sigma_{\delta}=\{\delta\} \times \Sigma$, which is totally geodesic in $\tilde{M}$. Then $D \tilde{M}$ is one of the manifolds constructed in Example (1) after Proposition 3.2 (with a reflection symmetry across a totally geodesic hypersurface). Let $\Sigma_{\delta}, S$ be equipped with the induced metric from $\tilde{g}, g$. Consider the map $\phi: \Sigma_{\delta} \rightarrow S$ given by $\phi(\delta, x)=\alpha_{x}(\delta)$, where $\alpha_{x}(s)$ is the geodesic in $\Omega$ starting from $x$ and perpendicular to $\Sigma$. It follows from the facts that $S$ is an embedded hypersurface and each $\alpha_{x}(s)$ is perpendicular to $S$ at $\alpha_{x}(\delta)$ that $\phi$ is a local isometry between $\Sigma_{\delta}$ and $S$. Since $\Sigma_{\delta}$ and $S$ are both compact, $\phi$ is a covering map. Let $p \in S$ and suppose there are three points $x, y, z$ in $\Sigma$ such that $\alpha_{x}(\delta)=\alpha_{y}(\delta)=\alpha_{z}(\delta)=p$. Then two of $\alpha_{x}^{\prime}(\delta), \alpha_{y}^{\prime}(\delta), \alpha_{z}^{\prime}(\delta)$ must be the same, as all of them are perpendicular to $S$ at $p$. Hence, $x, y$ and $z$ cannot be distinct. This implies $\phi$ is either injective or is a double cover. If $\phi$ is 
injective, then the map $x \mapsto \alpha_{x}(\delta)$ would be a diffeomorphism from $\Sigma$ to $S$. Hence $\Omega \backslash \overline{I \times \Sigma} \neq \emptyset$, which is a contradiction. We conclude that $\phi$ is a double cover. Hence $(\Omega, g)$ is one of the manifolds constructed in Example (2) after Proposition 3.2 .

Theorem 1.2 in Section 1 now follows directly from Theorem 4.1 , Theorem 4.2 and Proposition 3.1. Since the manifolds constructed in Example (2) after Proposition 3.2 are not simply connected, we also have the following:

Corollary 4.1. Let $(\Omega, g)$ be as in Theorem 4.2 satisfying Assumption A. If $\Omega$ is simply connected, then $(\Omega, g)$ is a geodesic ball in a simply connected space form.

\section{Appendix A. Estimates of GRAPHiCAL REPRESENTATION OF HYPERSURFACES}

In this appendix, we include some estimates concerning graphical representation of hypersurfaces with bounded second fundamental form in a general Riemannian manifold.

Let $\left(M^{n}, g\right)$ be a complete Riemannian manifold and $N$ be an immersed hypersurface in $M$. Assume $M$ and $N$ satisfy the following:

(a1) The curvature $R m$ and the covariant derivative $D R m$ of the curvature of $M$ are bounded.

(a2) The injectivity radius of $M$ is positive.

(a3) The norm of the second fundamental form of $N$ is uniformly bounded.

Let $a, b, c, \ldots$ denote indices ranging from 1 to $n$ and let $i, j, k, \ldots$ denote indices ranging from 1 to $n-1$. Let $B(p, r)$ denote a geodesic ball centered at $p \in M$ with radius $r$. The next lemma on normal coordinates in $(M, g)$ was proved in [7].

Lemma A.1. There exist constants $r>0$ and $C>0$ depending only on the bounds in (a1)-(a2) such that for any $p \in M$, the exponential map at $p$ is a diffeomorphism in $B(p, r)$, and if $\left(x^{1}, \ldots, x^{n}\right)$ are normal coordinates at $p$ in $B(p, r)$, then the components of the metric tensor $g$ in these coordinates satisfy

$$
\left|g_{a b}-\delta_{a b}\right|(x)+\left|\frac{\partial}{\partial x^{c}} g_{a b}\right|(x) \leq C|x| .
$$

Let $r>0$ be the constant in Lemma A.1. Let $p \in N$ and $\left\{x^{a}\right\}$ be normal coordinates in $B(p, r)$ such that $\left\{\frac{\partial}{\partial x^{i}}\right\}$ spans the tangent plane of $N$ at $p$. Let $x^{\prime}=\left(x^{1}, \ldots, x^{n-1}\right)$ and $\left|x^{\prime}\right|^{2}=\sum_{i=1}^{n-1}\left(x^{i}\right)^{2}$. We have

Lemma A.2. There exist $r>\rho_{0}>0$ independent of $p$ and a function $w=w\left(x^{\prime}\right)$ defined on $\left|x^{\prime}\right| \leq \rho_{0}$, such that $w(0)=0$ and $\left\{\left(x^{\prime}, w\left(x^{\prime}\right)\right)|| x^{\prime} \mid \leq \rho_{0}\right\}$ is part of $N$ passing through $p$. Moreover, there is a constant $C_{1}$ independent of $p$ such that $|w|+\left|\partial_{i} w\right|+\left|\partial_{i} \partial_{j} w\right| \leq C_{1}$ in $\left|x^{\prime}\right| \leq \rho_{0}$. Here $\partial_{i} w=\frac{\partial w}{\partial x^{i}}$ and $\partial_{i} \partial_{j} w=\frac{\partial^{2} w}{\partial x^{i} \partial x^{j}}$.

Proof. Let $w$ be a function defined near $x^{\prime}=0$ such that the graph $\left\{\left(x^{\prime}, w\right)\right\}$ is part of $N$ through $p$ and is inside $B(p, r)$. Suppose $\rho>0$ is such that the function $w\left(x^{\prime}\right)$ can be extended and defined in $\left|x^{\prime}\right| \leq \rho<r$ with $\left\{\left(x^{\prime}, w\right)\right\}$ being part of $N$ and inside $B(p, r)$. We want to estimate $\left|\partial_{i} w\right|$. Let $W(x)=w\left(x^{\prime}\right)-x^{n}$. The norm 
of the second fundamental form $A$ of $N$ is then given by

$$
\begin{aligned}
|A|^{2}= & \sum_{1 \leq a, b, c, d \leq n}\left(g^{a c}-\frac{W^{a} W^{c}}{|D W|^{2}}\right)\left(g^{b d}-\frac{W^{b} W^{d}}{|D W|^{2}}\right)\left(\frac{W_{a b}}{|D W|}\right)\left(\frac{W_{c d}}{|D W|}\right) \\
= & \sum_{1 \leq a, b, c, d \leq n} g^{a c} g^{b d}\left(\frac{W_{a b}}{|D W|}\right)\left(\frac{W_{c d}}{|D W|}\right) \\
& -2 \sum_{1 \leq a, b, c, d \leq n} g^{a c} \frac{W^{b} W^{d}}{|D W|^{2}}\left(\frac{W_{a b}}{|D W|}\right)\left(\frac{W_{c d}}{|D W|}\right) \\
& +\left(\sum_{1 \leq a, b \leq n} \frac{W^{a} W^{b} W_{a b}}{|D W|}\right)^{2},
\end{aligned}
$$

where $D W=W^{a} \frac{\partial}{\partial x^{a}}$ is the gradient of $W$ and $W_{a b} d x^{a} \otimes d x^{b}$ is the Hessian of $W$. (See [12.) Let

$$
I=\sum_{1 \leq a, b, c, d \leq n} g^{a c} g^{b d}\left(\frac{W_{a b}}{|D W|}\right)\left(\frac{W_{c d}}{|D W|}\right)
$$

and

$$
I I=\sum_{1 \leq a, b, c, d \leq n} g^{a c} \frac{W^{b} W^{d}}{|D W|^{2}}\left(\frac{W_{a b}}{|D W|}\right)\left(\frac{W_{c d}}{|D W|}\right) .
$$

Then $|A|^{2} \geq I-2 I I$. In the following, we always use $C$ to denote a constant depending only on the bounds in assumptions (a1)-(a3) and $n$, and use $f(\rho)$ to denote a function such that $|f(\rho)| \leq C \rho$. Both $C$ and $f(\rho)$ may vary from line to line.

Let $G(\rho)=\sup _{\left|x^{\prime}\right| \leq \rho}|\partial w|$, where $\partial w=\left(\partial_{1} w, \ldots, \partial_{n-1} w\right)$ and the norm is w.r.t. the Euclidean metric. We have the following estimates for $\left|x^{\prime}\right| \leq \rho$ :

$$
\begin{aligned}
&\left|w\left(x^{\prime}\right)\right| \leq G(\rho)\left|x^{\prime}\right|, \\
& W^{a}=g^{a b} W_{b} \\
&=W_{a}+\left(g^{a b}-\delta^{a b}\right) W_{b} \\
&=W_{a}+(1+G(\rho)) f(\rho),
\end{aligned}
$$

where $W_{a}=\frac{\partial W}{\partial x^{a}}$,

$$
\begin{gathered}
W_{i j}=\frac{\partial^{2} W}{\partial x^{i} \partial x^{j}}-\Gamma_{i j}^{a} W_{a} \\
=w_{i j}+(1+G(\rho)) f(\rho), \\
W_{a n}=\frac{\partial^{2} W}{\partial x^{a} \partial x^{n}}-\Gamma_{a n}^{b} W_{b} \\
=(1+G(\rho)) f(\rho) .
\end{gathered}
$$


Hence

$$
I=\sum_{1 \leq a, b \leq n} \frac{W_{a b}^{2}}{|D W|^{2}}+(1+G(\rho)) f(\rho) \sum_{1 \leq a, b \leq n} \frac{W_{a b}^{2}}{|D W|^{2}}
$$

$$
\begin{aligned}
I I & =\sum_{1 \leq a, b, d \leq n} \frac{W^{b} W^{d}}{|D W|^{2}}\left(\frac{W_{a b}}{|D W|}\right)\left(\frac{W_{a d}}{|D W|}\right) \\
& +\sum_{1 \leq a, b, c, d \leq n}\left(g^{a c}-\delta^{a c}\right) \frac{W^{b} W^{d}}{|D W|^{2}}\left(\frac{W_{a b}}{|D W|}\right)\left(\frac{W_{c d}}{|D W|}\right) \\
& =\frac{1}{|D W|^{4}}\left(\sum_{1 \leq i, j, k \leq n-1} W^{i} W^{j} W_{k i} W_{k j}\right)+(1+G(\rho)) f(\rho) \sum_{1 \leq a, b \leq n} \frac{W_{a b}^{2}+\left|W_{a b}\right|}{|D W|^{2}} \\
& =\frac{1}{|D W|^{4}} \sum_{1 \leq k \leq n-1}\left(\sum_{1 \leq i \leq n-1} W^{i} W_{k i}\right)^{2}+(1+G(\rho)) f(\rho) \sum_{1 \leq a, b \leq n} \frac{W_{a b}^{2}+\left|W_{a b}\right|}{|D W|^{2}}
\end{aligned}
$$

and hence

$$
\begin{aligned}
I I & \leq \frac{1}{|D W|^{4}} \sum_{1 \leq i \leq n-1}\left(W^{i}\right)^{2} \sum_{1 \leq k, i \leq n-1}\left(W_{k i}\right)^{2}+(1+G(\rho)) f(\rho) \sum_{1 \leq a, b \leq n} \frac{W_{a b}^{2}+\left|W_{a b}\right|}{|D W|^{2}} \\
& \leq \frac{2}{|D W|^{4}} \sum_{1 \leq i \leq n-1}\left(W^{i}\right)^{2} \sum_{1 \leq k, i \leq n-1}\left[w_{k i}^{2}+(1+G(\rho))^{2} f^{2}(\rho)\right] \\
& +(1+G(\rho)) f(\rho) \sum_{1 \leq a, b \leq n} \frac{W_{a b}^{2}+\left|W_{a b}\right|}{|D W|^{2}} \\
& \leq \frac{4}{|D W|^{4}} \sum_{1 \leq i \leq n-1}\left[w_{i}^{2}+(1+G(\rho))^{2} f^{2}(\rho)\right] \sum_{1 \leq k, i \leq n-1} w_{k i}^{2}+\frac{(1+G(\rho))^{2} f^{2}(\rho)}{|D W|^{2}} \\
& +(1+G(\rho)) f(\rho) \sum_{1 \leq a, b \leq n} \frac{W_{a b}^{2}}{|D W|^{2}} \\
& \leq \frac{8 n\left[G^{2}(\rho)\left(1+f^{2}(\rho)\right)+f^{2}(\rho)\right]}{|D W|^{4}} \sum_{1 \leq k, i \leq n-1} w_{k i}^{2}+\frac{(1+G(\rho))^{2} f^{2}(\rho)}{|D W|^{2}} \\
& +(1+G(\rho)) f(\rho) \sum_{1 \leq a, b \leq n} \frac{W_{a b}^{2}}{|D W|^{2}} .
\end{aligned}
$$

Therefore

$$
\begin{aligned}
|A|^{2} & \geq[1-(1+G(\rho)) f(\rho)] \frac{\sum_{1 \leq a, b \leq n} W_{a b}^{2}}{|D W|^{2}} \\
& -\frac{16 n\left[G^{2}(\rho)\left(1+f^{2}(\rho)\right)+f^{2}(\rho)\right]}{|D W|^{4}} \sum_{1 \leq k, i \leq n-1} w_{k i}^{2}-\frac{(1+G(\rho))^{2} f^{2}(\rho)}{|D W|^{2}} .
\end{aligned}
$$


Hence there exist $\alpha>0$ and $r>\rho_{1}>0$ depending only on the bounds in (a1) $-(\mathbf{a 3})$ and $n$ such that if $\rho \leq \rho_{1}$ and $G(\rho) \leq \alpha$, then

$$
\begin{gathered}
|(1+G(\rho)) f(\rho)| \leq \frac{1}{4}, \\
\left|16 n\left[G^{2}(\rho)\left(1+f^{2}(\rho)\right)+f^{2}(\rho)\right]\right| \leq \frac{1}{4}|D W|^{2},
\end{gathered}
$$

and so

$$
\sup _{\left|x^{\prime}\right| \leq \rho} \sum_{i j} w_{i j}^{2} \leq C\left(1+G^{2}(\rho)\right) .
$$

Since $w_{i}=0$ at $x^{\prime}=0$, we have

$$
G(\rho) \leq C \rho(1+G(\rho)),
$$

provided $\rho \leq \rho_{1}$ and $G(\rho) \leq \alpha$. Hence

$$
G(\rho) \leq \frac{C \rho}{1-C \rho}
$$

provided $\rho \leq \rho_{1}, G(\rho) \leq \alpha$ and $C \rho_{1}<1$. Now choose $\rho_{0}$ such that $0<\rho_{0}<\rho_{1}$, $C \rho_{0}<1$ and $\frac{C \rho_{0}}{1-C \rho_{0}} \leq \frac{\alpha}{2}$.

Let $\rho * \leq \rho_{0}$ be the supremum of $\rho$ such that $w$ can be extended on $\left|x^{\prime}\right| \leq \rho$ so that $\left(x^{\prime}, w\left(x^{\prime}\right)\right)$ is part of $N$ and such that $G(\rho) \leq \frac{\alpha}{2}$. We claim that $\rho^{*}=\rho_{0}$. Suppose $\rho^{*}<\rho_{0}$. Since $|\partial w| \leq \frac{\alpha}{2}$ in $\left|x^{\prime}\right|<\rho^{*}, w$ can be extended to $\left|x^{\prime}\right|=\rho^{*}$ and beyond. That is, we can find $\rho^{*}<\rho_{2} \leq \rho_{0}$ such that $w$ can be extended to $\left|x^{\prime}\right| \leq \rho_{2}<\rho_{0}$ such that $G\left(\rho_{2}\right) \leq \alpha$. We then have

$$
G\left(\rho_{2}\right) \leq \frac{C \rho_{2}}{1-C \rho_{2}} \leq \frac{\alpha}{2},
$$

which contradicts the definition of $\rho^{*}$.

\section{REFERENCES}

1. Bishop, R. L. and Crittenden, R. J., Geometry of manifolds, Pure and applied mathematics (Academic Press), 15, New York (1964). MR0169148 (29:6401)

2. Bishop, R. L. and O'Neill, B., Manifolds of negative curvature, Trans. Amer. Math. Soc. 145 (1969) 1-49. MR0251664(40:4891)

3. Cheng, S. Y., Eigenvalue comparison theorems and its geometric applications, Math. Z. 143 (1975), no. 3, 289-297. MR0378001 (51:14170)

4. Corvino, J., On the existence and stability of the Penrose compactification, Ann. Henri Poincaré 8 (2007), no. 3, 597-620. MR2329363 (2008e:53050)

5. Fischer, A. E. and Marsden, J. E., Deformations of the scalar curvature, Duke Math. J. 42 (1975), no. 3, 519-547. MR0380907 (52:1804)

6. Fischer-Colbrie, D. and Schoen, R., The structure of complete stable minimal surfaces in 3-manifolds of nonnegative scalar curvature, Comm. Pure Appl. Math. 33 (1980), no. 2, 199211. MR562550(81i:53044)

7. Hamilton, R. S., A compactness property for solutions of the Ricci flow, Amer. J. Math. 117 (1995), 545-572. MR:1333936 (96c:53056)

8. Kobayashi, O., A differential equation arising from scalar curvature function, J. Math. Soc. Japan 34 (1982), no. 4, 665-675. MR669275 (84a:53046)

9. Kobayashi, O. and Obata, M., Conformally-flatness and static space-time, Manifolds and Lie Groups (Notre Dame, Ind., 1980), Progr. Math., 14, Birkhäuser, Boston, MA (1981), 197-206. MR642858 (84c:53061)

10. Lafontaine, J., Conformal geometry from the Riemannian viewpoint, in Conformal geometry (Bonn, 1985/1986), 65-92, Aspects Math., E12, Vieweg, Braunschweig, 1988. MR979789 (90a:53022) 
11. Miao, P. and Tam, L.-F., On the volume functional of compact manifolds with boundary with constant scalar curvature, Calc. Var. Partial Differential Equations 36 (2009), no. 2, 141-171. MR2546025 (2010j:53054)

12. Schoen, R. and Yau, S.-T., Proof of the positive mass theorem. II, Comm. Math. Phys. 79 (1981), no. 2, 231-260. MR612249 (83i:83045)

School of Mathematical Sciences, Monash University, Victoria, 3800, Australia

E-mail address: Pengzi.Miao@sci.monash.edu.au

Current address: Department of Mathematics, University of Miami, Coral Gables, Florida 33124

E-mail address: pengzim@math.miami.edu

The Institute of Mathematical Sciences and Department of Mathematics, The Chinese University of Hong Kong, Shatin, Hong Kong, China

E-mail address: lftam@math.cuhk.edu.hk 